\title{
RELICS: Strong Lensing Analysis of MACS J0417.5-1154 and Predictions for Observing the Magnified High-redshift Universe with JWST
}

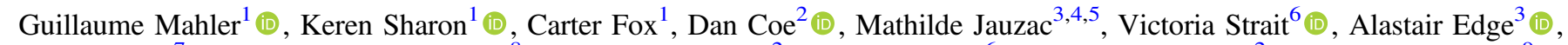
Ana Acebron $^{7}(1)$, Felipe Andrade-Santos ${ }^{8}\left(\mathbb{D}\right.$, Roberto J. Avila ${ }^{2}$, Maruša Bradač ${ }^{6}$, Larry D. Bradley ${ }^{2}\left(\mathbb{D}\right.$, Daniela Carrasco ${ }^{9}$ (i), Catherine Cerny ${ }^{10}$, Nathália Cibirka ${ }^{7}$ (1), Nicole G. Czakon ${ }^{11}$, William A. Dawson ${ }^{12}$ (1), Brenda L. Frye ${ }^{13}$, Austin T. Hoag ${ }^{6}$ (1), Kuang-Han Huang ${ }^{6}\left(\mathbb{D}\right.$, Traci L. Johnson ${ }^{14}$ (D) , Christine Jones $^{8}$, Shotaro Kikuchihara ${ }^{15}$, Daniel Lam ${ }^{16}(\mathbb{D})$, Rachael Livermore ${ }^{9,17}$ (D), Lorenzo Lovisari $^{8}$, Ramesh Mainali ${ }^{13}$ (1) , Sara Ogaz $^{2}$, Masami Ouchi ${ }^{15,18}$ (1), Rachel Paterno-Mahler ${ }^{1}$ (i) , Ian U. Roederer ${ }^{1,19}$ (1), Russell E. Ryan ${ }^{2}$, Brett Salmon ${ }^{2}$ (i), Irene Sendra-Server ${ }^{20}$, Daniel P. Stark ${ }^{13}$, Sune Toft ${ }^{21}$ (i), Michele Trenti ${ }^{9,17}$ (1), Keiichi Umetsu ${ }^{11}$ (1), Benedetta Vulcani ${ }^{9}$ (i), and Adi Zitrin ${ }^{7}$ (1)

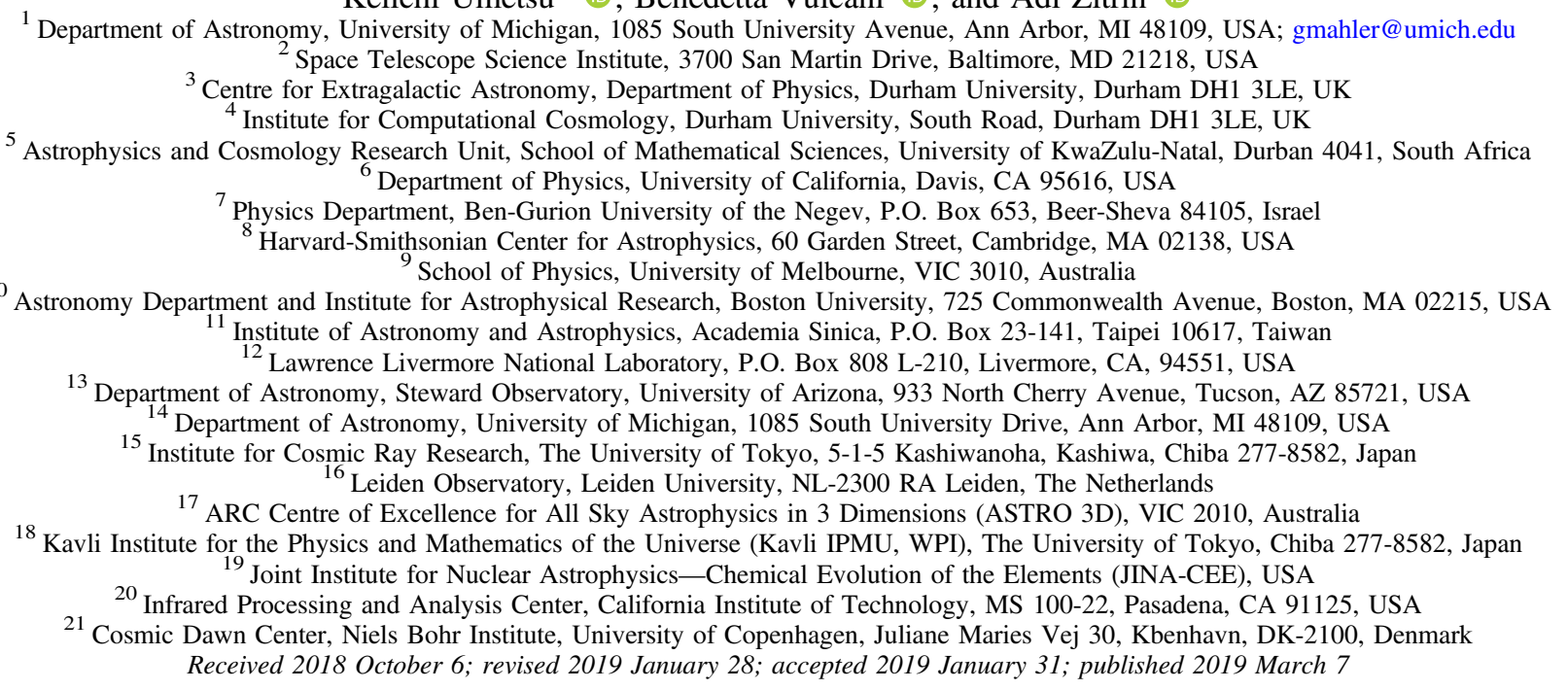

\begin{abstract}
Strong gravitational lensing by clusters of galaxies probes the mass distribution at the core of each cluster and magnifies the universe behind it. MACS J0417.5-1154 at $z=0.443$ is one of the most massive clusters known based on weak lensing, X-ray, and Sunyaev-Zel'dovich analyses. Here we compute a strong lens model of MACS J0417 based on Hubble Space Telescope imaging observations collected, in part, by the Reionization Lensing Cluster Survey (RELICS), and recently reported spectroscopic redshifts from the MUSE instrument on the Very Large Telescope (VLT). We measure an Einstein radius of $\theta_{E} \simeq 36^{\prime \prime}$ at $z=9$ and a mass projected within $200 \mathrm{kpc}$ of $M_{(200 \mathrm{kpc})}=1.78_{-0.03}^{+0.01} \times 10^{14} M_{\odot}$. Using this model, we measure a ratio between the mass attributed to cluster-member galaxy halos and the main cluster halo of order 1:100. We assess the probability to detect magnified high-redshift galaxies in the field of this cluster, both for comparison with RELICS HST results and as a prediction for the James Webb Space Telescope (JWST) Guaranteed Time Observations upcoming for this cluster. Our lensing analysis indicates that this cluster has similar lensing strength to other clusters in the RELICS program. Our lensing analysis predicts a detection of at least a few $z \sim 6-8$ galaxies behind this cluster, at odds with a recent analysis that yielded no such candidates in this field. Reliable strong lensing models are crucial for accurately predicting the intrinsic properties of lensed galaxies. As part of the RELICS program, our strong lensing model produced with the Lenstool parametric method is publicly available through the Mikulski Archive for Space Telescopes.
\end{abstract}

Key words: galaxies: clusters: individual (MACSJ0417.5-1154) - gravitational lensing: strong

\section{Introduction}

In our view of the history of the universe, the epoch of reionization remains the least well observed. During the first billion years, the universe was largely neutral. Half the intergalactic medium (IGM) in the universe was reionized by $z=8 \pm 1$ (Planck Collaboration et al. 2016a) and nearly completely by $z=6$. The end of reionization is evidenced by Gunn \& Peterson (1965) troughs (due to absorption by neutral intergalactic hydrogen) observed in $z>6$ quasar spectra, but not in spectra at $z<6$ (Becker et al. 2001, 2015; Djorgovski et al. 2001; Fan et al. 2006). Observing galaxies during the epoch of reionization remains a challenge today. They are much fainter due to their great distance and smaller sizes, and any Ly $\alpha$ emission is often scattered or absorbed by the surrounding neutral gas.

Strong lensing magnification by clusters of galaxies offers a privileged view of the high- $z$ universe. Several studies already highlight the high power of gravitational lenses to reveal 
objects that would have been inaccessible otherwise. Deep observations of Frontier Fields clusters (Lotz et al. 2017) were particularly important for probing the faint end of high-redshift luminosity functions and the galaxies most likely responsible for reionization (Atek et al. 2015, 2018; Livermore et al. 2017; Bouwens et al. 2017; Yue et al. 2018; Bhatawdekar et al. 2018; Ishigaki et al. 2018), as well as finding high-redshift candidates (e.g., a $z \sim 10$ galaxy from Oesch et al. 2018). The Cluster Lensing And Supernova survey with Hubble (CLASH; Postman et al. 2012) yielded $z \sim 6-11$ galaxies to be observed more brightly (Hashimoto et al. 2018; Zheng et al. 2012; Coe et al. 2013; Bradley et al. 2014). Even after these large surveys, many clusters had yet to be observed by the Hubble Space Telescope $(H S T)$ at near-infrared wavelengths $(1.0-1.7 \mu \mathrm{m})$ to search for distant galaxies.

MACS J0417.5-1154 (hereafter, MACS J0417) was discovered by the MAssive Cluster Survey (MACS; Ebeling et al. 2001) as part of the ROSAT (Voges et al. 1999) catalog of bright sources. MACS J0417 at $z=0.443$ is one of the most X-ray luminous clusters with a luminosity of $2.9 \times 10^{45} \mathrm{erg} \mathrm{s}^{-1}$ between 0.1 and $2.4 \mathrm{keV}$. Based on Chandra X-ray observations, Mann \& Ebeling (2012) report that the peak of the X-ray emission is centered on the primary brightest cluster galaxy (BCG) with a slight diffuse emission extended toward the second brightest galaxy in the cluster core. Dwarakanath et al. (2011), Parekh et al. (2017), and Sandhu et al. (2018) confirm this feature in the radio. Parekh et al. (2017) highlight the similarity in morphology to the clusters A2746 and 1E 0657-56 (the "Bullet cluster"), strengthening the hypothesis made by Mann \& Ebeling (2012) that MACS J0417 is a recent merger, probably oriented along the line of sight, or alternatively, caught close to a turnaround. The merging state of MACS J0417 is also confirmed in the analysis of Pandge et al. (2018). Recently, Botteon et al. (2018) discovered two new cold fronts indicating that substructure dynamics are at play in MACS J0417.

MACS J0417 was also detected by the Planck Early SunyaevZel'dovich (ESZ) catalog (Planck Collaboration et al. 2011), and with $M_{500}=(1.23 \pm 0.05) \times 10^{15} M_{\odot}$ had the fourth highest mass of all 1094 confirmed clusters with measured redshifts and mass estimates in the Planck PSZ2 catalog (Planck Collaboration et al. 2016b). Similarly, MACS J0417 has the third-highest mass $\left(M_{1500 \mathrm{kpc}}=(1.89 \pm 0.25) \times 10^{15} M_{\odot}\right)$ that was measured as a part of a weak lensing analysis of 27 clusters undertaken in the "Weighing the Giants" census (Applegate et al. 2014).

Based on all of these factors, MACS J0417 was included in the Reionization Lensing Cluster Survey (RELICS). RELICS is a large HST Treasury program, GO 14096 (PI: Coe), to observe 46 fields strongly lensed by 41 massive galaxy clusters. The primary goals of the program are to identify candidates of highredshift $(6<z<12)$ galaxies magnified by the foreground clusters (Salmon et al. 2017, 2018) with photometric redshifts estimated from multiband imaging with HST and Spitzer (PI: Bradač), and to better constrain luminosity functions at the epoch of reionization. Full details of the project will be described in a forthcoming publication (D. Coe et al. 2019, in preparation). Of particular interest is the potential to identify targets to be observed with the James Webb Space Telescope (JWST). To support this goal and increase the scientific impact of this program, strong lens models are being computed by the RELICS team (Cerny et al. 2018; Acebron et al. 2018a, 2018b; Cibirka et al. 2018; Paterno-Mahler et al. 2018; Salmon et al. 2018) and released to the scientific community via the Mikulski Archive for Space
Table 1

Details on the Observations of MACS J0417 Taken with the Hubble Space Telescope

\begin{tabular}{llcl}
\hline \hline Camera, filter & Exp. Time (s) & UT Date & Program \\
\hline ACS F435W & 2000.0 & 2016 Nov 30 & GO-14096 \\
WFC3/UVIS F606W & 5364.0 & 2011 Jan 20 & GO-12009 \\
WFC3/UVIS F606W & 1788.0 & 2011 Feb 28 & GO-12009 \\
ACS F814W & 1910.0 & 2010 Dec 10 & GO-12009 \\
WFC3/IR F105W & 705.9 & 2016 Dec 30 & GO-14096 \\
WFC3/IR F105W & 755.9 & 2017 Feb 10 & GO-14096 \\
WFC3/IR F125W & 380.9 & 2016 Dec 30 & GO-14096 \\
WFC3/IR F125W & 355.9 & 2017 Feb 11 & GO-14096 \\
WFC3/IR F140W & 380.9 & 2016 Dec 30 & GO-14096 \\
WFC3/IR F140W & 355.9 & 2017 Feb 10 & GO-14096 \\
WFC3/IR F160W & 1005.9 & 2016 Dec 30 & GO-14096 \\
WFC3/IR F160W & 1005.9 & 2017 Feb 11 & GO-14096 \\
\hline
\end{tabular}

Telescopes (MAST). ${ }^{22}$ The work presented here and in the companion paper, Jauzac et al. (2019), represent the first public strong lensing analyses on MACS J0417.5-1154.

The paper is organized as follows: In Section 2 we give an overview of the data. Section 3 details the strong lensing analysis, and the results are discussed in Section 4. In Section 5 we describe predictions for observing the high-redshift universe by current and future facilities. In Section 6 we summarize the main results of this work.

Throughout this paper we adopt a standard $\Lambda$-CDM cosmology with $\Omega_{m}=0.3, \Omega_{\Lambda}=0.7$ and $h=0.7$. All magnitudes are given in the AB system (Oke 1974).

\section{Data}

\subsection{Imaging}

\subsubsection{HST}

MACS J0417 was first observed by HST in Cycle 16, as part of a snapshot survey of the MACS clusters (SNAP 11103; PI: Ebeling) with the Wide Field Planetary Camera 2 (WFPC2) in the F606W and F814W bands. Deeper observations with the UVIS instrument on the Wide Field Camera 3 (WFC3) in the F606W band and with the Advanced Camera for Survey (ACS) in the F814W band were obtained in Cycle 17 as part of the Chandra proposal ID \#11800792 (joint with HST GO-12009; PI: von der Linden). It was then observed as part of the RELICS GO program with four filters on the WFC3-IR camera, F160W, F140W, F125W, and F105W; and F435W on ACS. Our analysis makes use of HST ACS and WFC3 imaging of MACS J0417, not the original WFPC2 shallow observations. Table 1 lists the dates and exposure times of the HST observations used in this work.

The ACS and WFC3 data were aligned to the same pixel frame and combined using standard procedures as described in Cerny et al. (2018). This work made use of images drizzled onto both 30 and 60 mas $^{-1}$, to take advantage of the full resolution capabilities of the WFC3/UVIS and ACS cameras, and proper sampling of the point-spread function. We provide fully reduced imaging data as service to the community, and they are publicly available as high-level data products on MAST.

\footnotetext{
${ }^{22}$ https://archive.stsci.edu/prepds/relics/
} 


\subsubsection{Spitzer}

The IRAC on board the Spitzer Space Telescope imaged MACSJ 0417 as part of the S-RELICS programme (SpitzerRELICS, PI: Bradač PI: Soifer). Observations reach $13 \mathrm{hr}$ of total exposure time in IRAC channels 1 and $2(3.6$ and $4.5 \mu \mathrm{m})$. The data reduction will be described in detail in V. Strait et al. (2019, in preparation); to create the mosaic images we use the MOSAICKER AND POINT SOURCE EXTRACTOR $\left(\right.$ MOPEX $\left.^{23}\right)$ and largely follow the process described in the IRAC Cookbook ${ }^{24}$ for the COSMOS medium-deep data.

The intracluster light subtraction and flux extraction are done using T-PHOT (Merlin et al. 2015), designed to perform PSF-matched, prior-based, multiwavelength photometry as described in Merlin et al. $(2015,2016)$. This is done by convolving cutouts from a high resolution image (in this case, F160W) using a low-resolution PSF transformation kernel that matches the F160W resolution to the IRAC (low-resolution) image. T-PHOT then fits a template to each source detected in F160W to best match the pixel values in the IRAC image. The IRAC fluxes are then combined with $H S T$ fluxes in catalogs.

\subsection{Spectroscopy}

\subsection{1. $L D S S 3$}

We obtained multislit spectroscopy of MACS J0417 with the upgraded Low Dispersion Survey Spectrograph (LDSS3-C) $)^{25}$ on the Magellan Clay telescope, on 2017 July 27 using the University of Michigan allocation (PI: Sharon). Two multislit masks were designed, with 1 ". 0 slits placed on multiple images of lensed galaxies at the highest priority, and the rest of the mask filled with background sources and cluster-member galaxies. Due to weather conditions, only one of the masks was observed, with three exposures of $1200 \mathrm{~s}$ each. The seeing ranged between $0 . " 5$ and $0 . ! 7$, with some clouds present during the observation. The data were obtained with the VPH-ALL grism $(4250 \AA<\lambda<10000 \AA)$ with spectral resolution $R=$ $450-1100$ across the wavelength range. The spectroscopic data were reduced using the standard procedures using the COSMOS data reduction package (Dressler et al. 2011; Oemler et al. 2017). We measured a spectroscopic redshift of $z_{\text {spec }}=$ 0.871 for image 1.3 $(\alpha=04: 17: 33.70, \delta=-11: 54: 39.70)$, based on [O II] $\lambda 3728$ and $\mathrm{H} \beta$ line emission. The data yielded a spectroscopic redshift for another background source $(\alpha=4: 17: 35.942, \delta=-11: 54: 59.29)$, at $z_{\text {spec }}=1.046$, from [O II] $\lambda 3728$, [O III] $\lambda 4959,5007$; however, this source is not multiply imaged and was not used as a constraint in the lens model (see Section 3).

A full description of the RELICS Magellan/LDSS3 followup results will be presented in a future paper (R. Mainali et al. 2019, in preparation).

\subsubsection{MUSE}

The field was observed with the Multi Unit Spectrographic Explorer (MUSE; Bacon et al. 2010) on 2017 December 12. The MUSE exposure was $3 \times 970 \mathrm{~s}$, or $2910 \mathrm{~s}$ in total, and was taken as part of ESO project 0100.A-0792(A) (PI: Edge). The

\footnotetext{
$\overline{23}$ http://irsa.ipac.caltech.edu/data/SPITZER/docs/dataanalysistools/tools/ mopex/

${ }^{24}$ http://irsa.ipac.caltech.edu/data/SPITZER/docs/dataanalysistools/ cookbook/

${ }^{25} \mathrm{http}$ // www.lco.cl/telescopes-information/magellan/instruments/ldss-3
}

data were reduced and spectra extracted as explained in the companion paper Jauzac et al. (2019). The MUSE field of view (FOV), $1^{\prime} \times 1^{\prime}$, is approximately centered on the BCG, and does not cover the full extent of the HST FOV. The MUSE spectral resolution is $R=1750-3750$ across the wavelength range 4800-9300 .

This work makes use of the spectroscopic redshifts measured for lensed galaxies reported in the companion paper by Jauzac et al. (2019) (Table 2). The MUSE observation confirms the redshift that was obtained with LDSS3 for image $1.3, z_{\text {spec }}=$ 0.871 , and spectroscopically confirms images 1.1 and 1.2 as counter images of the same system. Moreover, it reveals [O II] $\lambda 3728$ emission from a fourth image at the same redshift, buried in the light of the BCG. This fourth image is likely not a complete image; therefore, we did not use it as a constraint to model the cluster. The redshifts of system 2 and system 3 are both measured at $z_{\mathrm{spec}}=1.046$. The two systems correspond to two different galaxies separated by $\sim 140 \mathrm{kpc}$ in the source plane according to our modeling.

For images 4.2 and 4.1, the MUSE data are consistent with a low-confidence redshift of $z=3.10$. Due to the low confidence of this measurement, we do not use it as a constraint. A full description of the data and results related to other objects in the field are given in our companion paper by Jauzac et al. (2019).

\section{Gravitational Lensing Analysis}

\subsection{Methodology}

The lens model of MACS J0417 was computed using the public software Lenstool (Jullo et al. 2007), which is a parametric lens modeling algorithm that employs Markov Chain Monte Carlo (MCMC) analysis to explore the parameter space and identify the best-fit solution. The lens plane is modeled as a linear combination of several mass halos, each parameterized as a pseudo isothermal ellipsoidal mass distribution (PIEMD or dPIE; Elíasdóttir et al. 2007) with seven parameters: position $x, y$; ellipticity $\varepsilon$; position angle $\theta$; core radius $r_{c}$; cut radius $r_{\text {cut }}$; and normalization $\sigma_{0}$. The two radii parameters, $r_{c}$ and $r_{\text {cut }}$, define the region $r_{c} \lesssim r \lesssim r_{\text {cut }}$ in which the mass profile is isothermal; the mass density transitions smoothly, but drops rapidly beyond $r_{\text {cut }}$. The cluster mass distribution is typically dominated by cluster-scale and group-scale halos, whose parameters are set free. Galaxy-scale halos are placed at the observed positions of cluster-member galaxies, with positional parameters $(x, y, \theta, \varepsilon)$ fixed at the observed values of their light distribution as measured with SExtractor (Bertin \& Arnouts 1996) and the other parameters scaled with the luminosity of the galaxy in F814W, following scaling relations as described in Limousin et al. (2005). The normalization parameter of the scaling relation, $\sigma_{0}^{*}$, is a free parameter. The slope parameters and normalization of the three brightest galaxies are solved individually, and those are decoupled from the scaling relations of the other cluster members. The BCG is clearly bluer than the cluster red sequence due to ongoing star formation (Green et al. 2016) and therefore is not expected to follow the same scaling relation (Postman et al. 2012). The other two galaxies dominate the subgroups at the north of the FOV, and by leaving their parameters free we allow for a larger contribution of underlying dark matter (DM) halo in this region.

An alternative approach would be to model these two galaxies separately and add two other group-scale halos to 
Table 2

List of Lensing Constraints

\begin{tabular}{|c|c|c|c|c|c|c|c|c|}
\hline ID & $\begin{array}{l}\text { R.A. } \\
\text { J2000 }\end{array}$ & $\begin{array}{l}\text { Decl. } \\
\text { J2000 }\end{array}$ & $z_{\text {spec }}$ & $\begin{array}{l}z_{\text {model }} \\
\text { silver }\end{array}$ & $\begin{array}{c}\text { rms (") } \\
\text { silver }\end{array}$ & $\begin{array}{l}z_{\text {model }} \\
\text { bronze }\end{array}$ & $\begin{array}{l}\text { rms (") } \\
\text { bronze }\end{array}$ & Classification \\
\hline $\begin{array}{l}1 \mathrm{a} .1 \\
1 \mathrm{a} .2 \\
1 \mathrm{a} .3 \\
1 \mathrm{~b} .1 \\
1 \mathrm{~b} .2 \\
1 \mathrm{~b} .3 \\
1 \mathrm{c} .2 \\
1 \mathrm{c} .1 \\
1 \mathrm{c} .3\end{array}$ & $\begin{array}{l}64.396158 \\
64.394310 \\
64.390348 \\
64.396081 \\
64.394729 \\
64.390299 \\
64.394371 \\
64.396364 \\
64.390488\end{array}$ & $\begin{array}{l}-11.906760 \\
-11.907136 \\
-11.910864 \\
-11.907255 \\
-11.907583 \\
-11.911274 \\
-11.907409 \\
-11.906983 \\
-11.911052\end{array}$ & $0.8710^{\mathrm{a}}$ & $\cdots$ & $\begin{array}{l}0.07 \\
0.16 \\
0.09 \\
0.32 \\
0.43 \\
0.09 \\
0.26 \\
0.13 \\
0.10\end{array}$ & $\cdots$ & $\begin{array}{l}0.10 \\
0.22 \\
0.09 \\
0.36 \\
0.53 \\
0.13 \\
0.35 \\
0.17 \\
0.12\end{array}$ & gold \\
\hline $\begin{array}{l}2 \mathrm{a} .1 \\
2 \mathrm{a} .2 \\
2 \mathrm{a} .3 \\
2 \mathrm{~b} .1 \\
2 \mathrm{~b} .2 \\
2 \mathrm{~b} .3 \\
2 \mathrm{c} .1 \\
2 \mathrm{c} .2 \\
2 \mathrm{c} .3\end{array}$ & $\begin{array}{l}64.399096 \\
64.395567 \\
64.391371 \\
64.399000 \\
64.395821 \\
64.391262 \\
64.399004 \\
64.395954 \\
64.391300\end{array}$ & $\begin{array}{l}-11.906369 \\
-11.911182 \\
-11.912074 \\
-11.906633 \\
-11.911226 \\
-11.912324 \\
-11.906855 \\
-11.911299 \\
-11.912493\end{array}$ & $1.0460^{\mathrm{b}}$ & $\cdots$ & $\begin{array}{l}0.41 \\
0.42 \\
0.18 \\
0.50 \\
0.47 \\
0.24 \\
0.49 \\
0.48 \\
0.24\end{array}$ & $\cdots$ & $\begin{array}{l}0.47 \\
0.45 \\
0.24 \\
0.57 \\
0.48 \\
0.30 \\
0.56 \\
0.49 \\
0.30\end{array}$ & gold \\
\hline $\begin{array}{l}3.1 \\
3.2 \\
3.3\end{array}$ & $\begin{array}{l}64.393180 \\
64.390026 \\
64.388304\end{array}$ & $\begin{array}{l}-11.901537 \\
-11.903434 \\
-11.905013\end{array}$ & $1.0460^{\mathrm{b}}$ & $\cdots$ & $\begin{array}{l}0.72 \\
0.81 \\
0.30\end{array}$ & $\cdots$ & $\begin{array}{l}0.59 \\
0.89 \\
0.56\end{array}$ & gold \\
\hline $\begin{array}{l}4.1 \\
4.2 \\
4.3\end{array}$ & $\begin{array}{l}64.399521 \\
64.398529 \\
64.386095\end{array}$ & $\begin{array}{l}-11.907479 \\
-11.909839 \\
-11.915359\end{array}$ & $\cdots$ & $2.26_{-0.08}^{+0.08}$ & $\begin{array}{l}0.26 \\
0.65 \\
0.60\end{array}$ & $2.33_{-0.07}^{+0.07}$ & $\begin{array}{l}0.50 \\
0.47 \\
0.34\end{array}$ & silver \\
\hline $\begin{array}{l}5.1 \\
5.2 \\
5.3\end{array}$ & $\begin{array}{l}64.379941 \\
64.382370 \\
64.388438\end{array}$ & $\begin{array}{l}-11.897906 \\
-11.896413 \\
-11.891630\end{array}$ & $\cdots$ & $2.27_{-0.14}^{+0.11}$ & $\begin{array}{l}0.24 \\
0.27 \\
0.51\end{array}$ & $2.25_{-0.07}^{+0.09}$ & $\begin{array}{l}0.20 \\
0.25 \\
0.49\end{array}$ & silver \\
\hline $\begin{array}{l}6.1 \\
6.2 \\
6.3 \\
\end{array}$ & $\begin{array}{l}64.379991 \\
64.381808 \\
64.388558 \\
\end{array}$ & $\begin{array}{r}-11.897349 \\
-11.896390 \\
-11.891170 \\
\end{array}$ & $\cdots$ & $2.34_{-0.17}^{+0.11}$ & $\begin{array}{l}0.21 \\
0.18 \\
0.46 \\
\end{array}$ & $2.27_{-0.06}^{+0.09}$ & $\begin{array}{l}0.24 \\
0.15 \\
0.42 \\
\end{array}$ & silver \\
\hline $\begin{array}{l}7.1 \\
7.2\end{array}$ & $\begin{array}{l}64.394933 \\
64.388688\end{array}$ & $\begin{array}{l}-11.897423 \\
-11.900546\end{array}$ & $\cdots$ & $\mathrm{d}^{\mathrm{d}} 2.09_{-0.05}^{+0.05}$ & $\begin{array}{l}{ }^{\mathrm{d}} 0.18 \\
{ }^{\mathrm{d}} 0.26\end{array}$ & $2.09_{-0.08}^{+0.12}$ & $\begin{array}{l}0.32 \\
0.28\end{array}$ & bronze \\
\hline $\begin{array}{l}8.1 \\
8.2\end{array}$ & $\begin{array}{l}64.388372 \\
64.386885\end{array}$ & $\begin{array}{l}-11.894492 \\
-11.895489\end{array}$ & $\cdots$ & $2.39_{-0.12}^{+0.14}$ & $\begin{array}{l}0.12 \\
0.14\end{array}$ & $2.35_{-0.09}^{+0.12}$ & $\begin{array}{l}0.06 \\
0.04\end{array}$ & silver \\
\hline $\begin{array}{l}9.1 \\
9.2 \\
\end{array}$ & $\begin{array}{l}64.382068 \\
64.382338 \\
\end{array}$ & $\begin{array}{r}-11.899994 \\
-11.899779 \\
\end{array}$ & $\cdots$ & $5.97_{-0.20}^{+0.01}$ & $\begin{array}{l}0.35 \\
0.22 \\
\end{array}$ & $5.52_{-0.31}^{+0.10}$ & $\begin{array}{l}0.43 \\
0.24 \\
\end{array}$ & silver \\
\hline $\begin{array}{l}10.1 \\
10.2 \\
10.3\end{array}$ & $\begin{array}{l}64.398397 \\
64.397785 \\
64.385000\end{array}$ & $\begin{array}{l}-11.907143 \\
-11.909114 \\
-11.915063\end{array}$ & $\cdots$ & $\begin{array}{c}2.02_{-0.10}^{+0.14} \\
\mathrm{~d}^{\mathrm{d}} 2.34_{-0.04}^{+0.05}\end{array}$ & $\begin{array}{l}0.28 \\
0.37 \\
{ }^{\mathrm{d}} 0.30\end{array}$ & $2.33_{-0.09}^{+0.07}$ & $\begin{array}{l}0.43 \\
0.76 \\
0.33\end{array}$ & $\begin{array}{c}\text { silver } \\
\text { bronze }^{\mathrm{c}}\end{array}$ \\
\hline $\begin{array}{l}11.1 \\
11.2\end{array}$ & $\begin{array}{l}64.401544 \\
64.399708\end{array}$ & $\begin{array}{l}-11.918912 \\
-11.920099\end{array}$ & $\cdots$ & $3.47_{-0.31}^{+0.36}$ & $\begin{array}{l}0.18 \\
0.30\end{array}$ & $3.18_{-0.11}^{+0.25}$ & $\begin{array}{l}0.09 \\
0.41\end{array}$ & silver \\
\hline $\begin{array}{l}12.1 \\
12.2 \\
12.3 \\
\end{array}$ & $\begin{array}{l}64.396902 \\
64.388640 \\
64.383172 \\
\end{array}$ & $\begin{array}{l}-11.897085 \\
-11.901300 \\
-11.906519 \\
\end{array}$ & $\cdots$ & $2.84_{-0.13}^{+0.13}$ & $\begin{array}{l}0.42 \\
0.77 \\
0.26 \\
\end{array}$ & $2.81_{-0.14}^{+0.16}$ & $\begin{array}{l}0.34 \\
0.62 \\
0.19 \\
\end{array}$ & silver \\
\hline $\begin{array}{l}13.1 \\
13.2 \\
13.3\end{array}$ & $\begin{array}{l}64.397312 \\
64.388420 \\
64.383499\end{array}$ & $\begin{array}{l}-11.897068 \\
-11.901684 \\
-11.906446\end{array}$ & $\cdots$ & $2.89_{-0.13}^{+0.15}$ & $\begin{array}{l}0.36 \\
0.73 \\
0.28\end{array}$ & $2.85_{-0.17}^{+0.14}$ & $\begin{array}{l}0.32 \\
0.58 \\
0.17\end{array}$ & silver \\
\hline $\begin{array}{l}14.1 \\
14.2 \\
\end{array}$ & $\begin{array}{l}64.382335 \\
64.382972 \\
\end{array}$ & $\begin{array}{l}-11.900359 \\
-11.899802 \\
\end{array}$ & $\cdots$ & ${ }^{\mathrm{d}} 4.40_{-0.21}^{+0.43}$ & $\begin{array}{l}{ }^{\mathrm{d}} 0.03 \\
{ }^{\mathrm{d}} 0.03\end{array}$ & $4.43_{-0.39}^{+0.29}$ & $\begin{array}{l}0.10 \\
0.12 \\
\end{array}$ & bronze \\
\hline $\begin{array}{l}15.1 \\
15.2 \\
15.3\end{array}$ & $\begin{array}{l}64.378193 \\
64.381890 \\
64.385361\end{array}$ & $\begin{array}{l}-11.894510 \\
-11.892331 \\
-11.890071\end{array}$ & $\cdots$ & $2.11_{-0.16}^{+0.16}$ & $\begin{array}{l}0.28 \\
0.29 \\
0.15\end{array}$ & $2.09_{-0.08}^{+0.09}$ & $\begin{array}{l}0.15 \\
0.20 \\
0.04\end{array}$ & silver \\
\hline
\end{tabular}


Table 2

(Continued)

\begin{tabular}{|c|c|c|c|c|c|c|c|c|}
\hline ID & $\begin{array}{l}\text { R.A. } \\
\text { J2000 }\end{array}$ & $\begin{array}{l}\text { Decl. } \\
\text { J2000 }\end{array}$ & $z_{\text {spec }}$ & $\begin{array}{l}z_{\text {model }} \\
\text { silver }\end{array}$ & $\begin{array}{c}\mathrm{rms}\left({ }^{(\prime)}\right) \\
\text { silver }\end{array}$ & $\begin{array}{c}z_{\text {model }} \\
\text { bronze }\end{array}$ & $\begin{array}{l}\text { rms }(") \\
\text { bronze }\end{array}$ & Classification \\
\hline 16.1 & 64.385599 & -11.886984 & $\cdots$ & $4.50_{-0.96}^{+1.91}$ & 0.16 & $4.66_{-0.33}^{+0.58}$ & 0.16 & silver \\
\hline 16.2 & 64.380143 & -11.888425 & & & 0.31 & & 0.26 & \\
\hline 16.3 & 64.376525 & -11.892540 & & & 0.02 & & 0.30 & \\
\hline 17.1 & 64.388212 & -11.895269 & $\ldots$ & $2.30_{-0.11}^{+0.10}$ & 0.21 & $2.16_{-0.06}^{+0.14}$ & 0.10 & silver \\
\hline 17.2 & 64.387833 & -11.895536 & & & 0.24 & & 0.11 & \\
\hline
\end{tabular}

Notes. R.A. and decl. refer to R.A. and decl. of the constraints position. $z_{\text {spec }}$ refers to the spectroscopic constraints when available; references for the spectroscopic redshifts are given in the table footnotes. $z_{\text {model }}$ indicates the best-fit redshift estimates resulting from the "silver" and "bronze" lens models with their respective statistical uncertainties. The rms is the difference between the observed position of a multiple image and the predicted position from the barycenter of our best-fit model in the image plane given in arcseconds. The classification scheme is discussed in Section 3.2.

${ }^{a}$ Spectroscopic redshift from Magellan/LDSS3 (this work) and confirmed by MUSE in Jauzac et al. (2019).

${ }^{\mathrm{b}}$ Spectroscopic redshifts from MUSE presented in Jauzac et al. (2019). Sources 2 and 3 are at the same redshift. These galaxies are separated by $\sim 140 \mathrm{kpc}$ in the source plane.

${ }^{c}$ In system 10, images 10.1 and 10.2 are classified as silver and 10.3 is classified as bronze. Image 10.3 was therefore not included in the "silver" model.

$\mathrm{d}$ These redshifts and rms values are computed using the best-fit model computed with silver constraints fixed, while only the redshifts of those systems are being optimized.

model their DM component, allowing flexibility in the position of the underlying total potential, as is done in our companion paper by Jauzac et al. (2019).

Following the red sequence technique of Gladders \& Yee (2000), we select cluster members from a color-magnitude diagram using F606W-F814W versus F814W, bracketing appropriately the $4000 \AA$ break, which is a typical feature observed in elliptical galaxies. We selected galaxies down to 24 mag, which corresponds approximately to $0.01 L^{*}$ at redshift 0.44 resulting in 177 galaxies identified as cluster members in total. The magnitude is referred to the SExtractor parameters MAG_AUTO as defined in Sextractor (Bertin \& Arnouts 1996).

The lens model is constrained with sets of multiple images, identified in the HST imaging data and classified as described below. The position of each image is used as a constraint. Where substructure is clearly identified and can be robustly matched between images, we use multiple emission knots in each image, which indirectly constrains the relative magnification between images. We refrain from over-weighting systems by limiting the number of emission knots used in any single image to four.

Where known, spectroscopic redshifts are used as fixed redshift constraints. These are available for systems 1, 2, and 3 . Most of the other systems have photometric redshifts from the RELICS analysis. However, following Cerny et al. (2018) and Johnson \& Sharon (2016), who studied the effects of redshift accuracy on the lens model, the redshifts of systems with no spectroscopic redshifts $\left(z_{\text {spec }}\right)$ are left as free parameters with broad limits, to avoid biases due to photometric redshift $\left(z_{\text {phot }}\right)$ outliers. We check the model-predicted source redshifts against the photometric redshift in Section 4.1 as an independent confirmation that the model is not converging onto a completely wrong solution (see the discussion in Cerny et al. 2018).

\subsection{Lensing Constraints}

We identify 57 images of 17 systems that are used as constraints and seven candidates of strongly lensed images. Following the Hubble Frontier Fields ranking process, we classify the observed lensed images into three categories: gold, silver, and bronze. The gold category includes robustly identified multiply imaged systems with a measured spectroscopic redshift; three systems fall into this category. The silver classification is given to multiply imaged systems that are reliably identified as such by morphology, surface brightness, and lensing symmetry; 12 systems fall into this category. Images that have less robust identification, or would not be identified as counter images without an accurate lens model, were put in the bronze category and not used as constraints in our fiducial (silver) model. All systems are shown in Figure 1, and their coordinates, redshifts, and ranking, are tabulated in Table 2. We note that system 4 has a possible redshift of 3.1 from MUSE; however, it is based on low-confidence features. We choose to not include the redshift as a constraint in the model, as if it is incorrect the redshift might bias the model as was shown by, e.g., Jauzac et al. (2015), Johnson \& Sharon (2016), Cerny et al. (2018), and Remolina González et al. (2018).

We identify several other strong lensing features in the field, which, at the depth of the data in hand, are not deemed reliable enough to be used as constraints. We list these candidates in this paper for completeness. All the candidates are presented in Figure 1, and their coordinates are tabulated in Table 4 in the Appendix A.

\subsection{Mass Model Components}

As described in Section 3.1, and as is typical for parametric lens modeling algorithms, the lens plane is described by a combination of several DM halos whose parameters are allowed to vary, with contribution from galaxy-scale halos that follow scaling relations. The lens model of MACS J0417 includes four "free" DM halos, all parameterized as PIEMDs. The dominant component is a cluster-scale halo, whose parameters are all allowed to vary, with the exception of the truncation radius $r_{\text {cut }}$ that extends beyond the strong lensing regime and cannot be constrained by the strong lensing evidence. Three other halos are placed on the three BCGs, with positional parameters $(x, y, \varepsilon$, and $\theta)$ following their light distribution, and the other parameters set free. We emphasize that the halos placed on these galaxies are not to be considered strictly galaxy halos. The model cannot disentangle the DM halo in which the galaxy is embedded from the underlying DM halo of the cluster or group. 
Table 3

Candidate Lens Models and Best-fit Parameters

\begin{tabular}{|c|c|c|c|c|c|c|c|c|}
\hline $\begin{array}{l}\text { Model Name } \\
\text { (Fit statistics) }\end{array}$ & $\begin{array}{c}\text { Component } \\
\ldots\end{array}$ & $\begin{array}{l}\Delta \alpha^{\mathrm{a}} \\
\left({ }^{\prime \prime}\right)\end{array}$ & $\begin{array}{l}\Delta \delta^{\mathrm{a}} \\
\left({ }^{\prime \prime}\right)\end{array}$ & $\varepsilon^{\mathrm{b}}$ & $\begin{array}{c}\theta \\
(\operatorname{deg})\end{array}$ & $\begin{array}{c}\sigma_{0} \\
\left(\mathrm{~km} \mathrm{~s}^{-1}\right)\end{array}$ & $\begin{array}{c}r_{\mathrm{cut}} \\
(\mathrm{kpc})\end{array}$ & $\begin{array}{l}r_{\text {core }} \\
(\mathrm{kpc})\end{array}$ \\
\hline Silver constraints & DM & $6.2_{-0.8}^{+1.0}$ & $9.1_{-0.9}^{+1.3}$ & $0.78_{-0.01}^{+0.01}$ & $54.2_{-0.3}^{+0.2}$ & $1299.1_{-21.4}^{+16.9}$ & [1500.0] & $32.8_{-1.2}^{+1.4}$ \\
\hline $\mathrm{rms}=0 ! " 37$ & 1stBCG & {$[0.0]$} & {$[0.1]$} & [0.64] & {$[60.5]$} & $587.5_{-9.3}^{+2.7}$ & $28.5_{-3.4}^{+13.2}$ & $1.2_{-0.2}^{+0.2}$ \\
\hline \multirow[t]{3}{*}{$\mathrm{BIC}=150$} & 2ndBCG & {$[47.8]$} & [69.6] & {$[0.35]$} & [74.1] & $367.5_{-18.5}^{+14.7}$ & $70.6_{-11.5}^{+18.2}$ & $0.5_{-0.3}^{+0.4}$ \\
\hline & 3rdBCG & [46.9] & {$[48.4]$} & {$[0.16]$} & {$[50.6]$} & $256.5_{-13.9}^{+9.9}$ & $74.9_{-24.1}^{+17.3}$ & $0.2_{-0.1}^{+0.7}$ \\
\hline & $L^{*}$ Galaxy & $\ldots$ & $\ldots$ & $\ldots$ & $\cdots$ & $119.8_{-12.0}^{+9.7}$ & $\cdots$ & $\cdots$ \\
\hline $\mathrm{rms}=0$ !" 37 & $1 \mathrm{stBCG}$ & {$[0.0]$} & {$[0.1]$} & {$[0.64]$} & {$[60.5]$} & $597.0_{-8.7}^{+3.5}$ & $41.0_{-16.3}^{+20.9}$ & $1.6_{-0.2}^{+0.2}$ \\
\hline \multirow[t]{4}{*}{$\mathrm{BIC}=164$} & 2ndBCG & [47.8] & [69.6] & {$[0.35]$} & [74.1] & $394.8_{-15.1}^{+4.9}$ & $55.4_{-13.9}^{+13.9}$ & $1.0_{-0.3}^{+0.2}$ \\
\hline & 3rdBCG & [46.9] & [48.4] & {$[0.16]$} & {$[50.6]$} & $267.0_{-8.0}^{+17.2}$ & $50.9_{-10.6}^{+21.6}$ & $0.7_{-0.2}^{+0.7}$ \\
\hline & $L^{*}$ Galaxy & $\cdots$ & $\cdots$ & $\cdots$ & $\ldots$ & $116.0_{-17.1}^{+9.9}$ & $\cdots$ & $\cdots$ \\
\hline & $\cdots$ & $\cdots$ & $\cdots$ & $\cdots$ & $\cdots$ & $\cdots$ & $\cdots$ & $\cdots$ \\
\hline & 2ndBCG & {$[47.8]$} & [69.6] & {$[0.35]$} & [74.1] & $379.7_{-8.8}^{+16.0}$ & $62.1_{-8.8}^{+16.7}$ & $0.5_{-0.1}^{+0.2}$ \\
\hline & 3rdBCG & [46.9] & [48.4] & {$[0.16]$} & {$[50.6]$} & $298.9_{-15.5}^{+12.7}$ & $120.7_{-21.3}^{+2.5}$ & $1.5_{-1.1}^{+0.0}$ \\
\hline & $L^{*}$ Galaxy & $\cdots$ & $\ldots$ & $\cdots$ & $\ldots$ & $94.4_{-11.9}^{+10.8}$ & $\cdots$ & $\cdots$ \\
\hline$\cdots$ & $\cdots$ & $\cdots$ & $\cdots$ & $\cdots$ & $\cdots$ & $\cdots$ & $\cdots$ & $\cdots$ \\
\hline
\end{tabular}

Notes.

${ }^{\text {a }} \Delta \alpha$ and $\Delta \delta$ are measured relative to the reference coordinate point: ( $\left.\alpha=04: 17: 34.6925, \delta=-11: 54: 31.9356\right)$.

${ }^{\mathrm{b}}$ Ellipticity $(\varepsilon)$ is defined to be $\left(a^{2}-b^{2}\right) /\left(a^{2}+b^{2}\right)$, where $a$ and $b$ are the semimajor and semiminor axes of the ellipse.

${ }^{c}$ Quantities in brackets are fixed parameters.

Table 4

List of Candidate Lensed Galaxies

\begin{tabular}{lcc}
\hline \hline ID & R.A. & Decl. \\
& J2000 & J2000 \\
\hline c18.1 & 64.40084583 & -11.91028778 \\
c18.2 & 64.40074167 & -11.91053000 \\
\hline $\mathrm{c} 19.1$ & 64.38539166 & -11.90097528 \\
$\mathrm{c} 19.2$ & 64.38445834 & -11.90156944 \\
\hline $\mathrm{c} 20.1$ & 64.39867042 & -11.91895096 \\
\hline $\mathrm{c} 21.1$ & 64.40059584 & -11.91285222 \\
$\mathrm{c} 21.2$ & 64.39874584 & -11.91455333 \\
\hline $\mathrm{c} 22.1$ & 64.39631667 & -11.91718722 \\
$\mathrm{c} 22.2$ & 64.39631667 & -11.91718722 \\
\hline $\mathrm{c} 23.1$ & 64.38672916 & -11.90686278 \\
$\mathrm{c} 23.2$ & 64.38670763 & -11.90698944 \\
\hline $\mathrm{c} 24.1$ & 64.39396249 & -11.91067667 \\
$\mathrm{c} 24.2$ & 64.39380000 & -11.91072361 \\
\hline
\end{tabular}

Table 3 lists the best-fit parameters of each halo, for several lens models. The "Silver" model uses as constraints the gold and silver arcs. The "Bronze" model uses gold, silver, and bronze constraints. We describe the third test model, labeled "Bridge," below.

As can be visually gleaned from the distribution of galaxies (Figure 1), the cluster core is fairly elongated, with the second and third brightest galaxies significantly separated in projection from the BCG. In the X-ray, Mann \& Ebeling (2012) and Parekh et al. (2017) report extended emission elongated in the SE-NW direction. We, therefore, compute an additional lens model that includes a fifth PIEMD DM halo, forming a mass "bridge" between the central and NW components. We test this hypothesis using the gold +silver list of constraints. The fifth halo is free to vary between the BCG and the NW component. The core radius of the potential is intentionally free to vary up to a high value $(300 \mathrm{kpc})$ to allow a possible flat profile. The cut radius is fixed to a $1.5 \mathrm{Mpc}$ as the main DM halo potential

We quantitatively compare the quality of the three lens models using two criteria. The first one is the rms, which describes how well the model reproduces the image-plane positions of the constraints. The second one is the Bayesian Information Criterion (BIC; introduced by Schwarz 1978), which is a statistical measurement based on the model likelihood $\mathcal{L}$, penalized by the number of free parameters $k$ and the number of constraints $n$ :

$$
\mathrm{BIC}=-2 \times \log (\mathcal{L})+k \times \log (n) .
$$

The rms gives a good indication of the global distance between the predicted image positions compared to the observed ones; thus for a fixed number of constraints a low rms generally implies a better model. The BIC quantifies an improvement in the model likelihood while taking into account a possible difference in the number of parameters and/or constraints between models. Thus a favorable model will be one with the best likelihood while keeping the lowest BIC value possible. Such criteria were used in previous analyses (Lagattuta et al. 2017; Jauzac et al. 2018; 


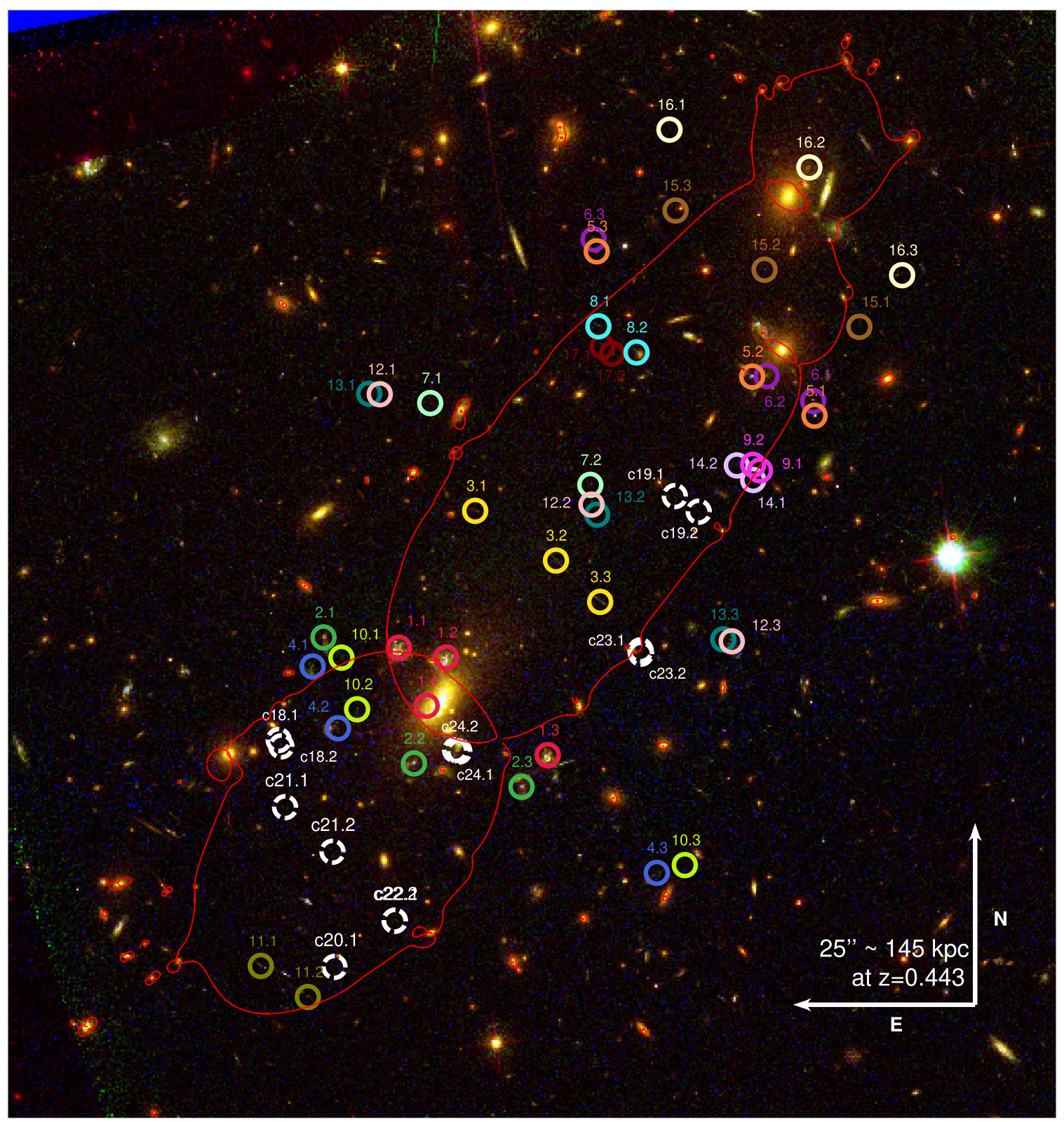

Figure 1. Composite color image of MACS J0417 created from HST imaging in ACS F814W (red), WFC3/UVIS F606W (green), and ACS F435W (blue). Secure multiply imaged galaxies (gold, silver, and bronze) are labeled with colored circles, color-coded by system. The white dashed circles label candidate images that were not used as constraints. The red line marks the location of the critical curve for a source at $z=9$.

Mahler et al. 2018) to compare different variation models for a single cluster. The rms of the "Bridge" model is slightly better (0"'36) compared to the fiducial model (0!"37). However, the BIC shows an opposite trend when comparing the two models. We interpret a higher BIC value for the "Bridge" model as an over-fit of the model compared to a model without the bridge. In other words, the model does not improve enough to justify the addition of new parameters. Similar statistical analyses were made in other studies, e.g., using a discrimination by the evidence (Limousin et al. 2010), other likelihood penalization: Akaike Information Criterion (Acebron et al. 2017) or a combination of a large number of indicators (Jauzac et al. 2018).

We compare the mass distribution between the models and plot their mass contours in Figure 2. The difference between the two models is most notably the southeast region of the cluster. While the BCG area is well-constrained by systems surrounding the BCG, there is only one system with two images farther out. A confirmation of some of the lensed galaxy candidates with deeper observations would better constrain this region.

\section{Discussion of Lens Model Results}

The spectroscopic capabilities of MUSE allow us to detect a central image for system 1 buried in the light of the BCG. Our model predicts a radial pair at this location; however, only a single peak of emission is visible. We interpret that as the likely result of the source-plane caustic bisecting the galaxy in the source plane, resulting in a merging pair configuration where only a small fraction of the source galaxy is lensed into these positions. A more detailed analysis of the lensing configuration of this galaxy is presented in the companion paper by Jauzac et al. (2019). 


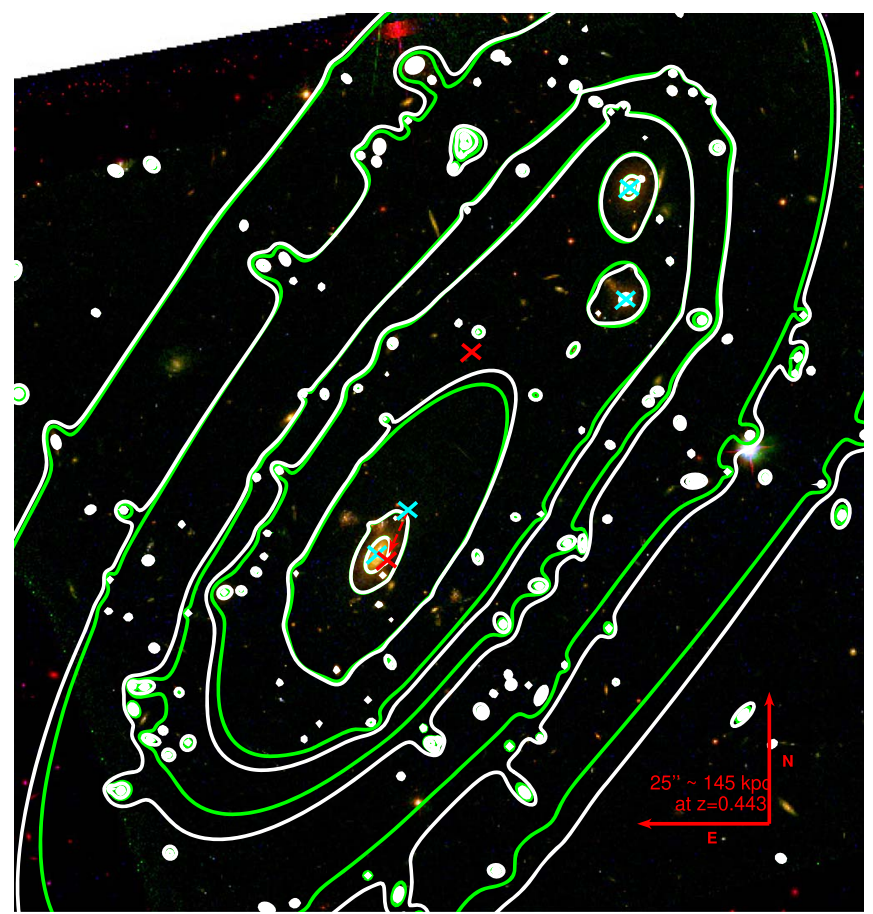

Figure 2. Cyan crosses show the position of all the individual DM potentials for our fiducial model. The top red cross shows the position of the center of the bridge potential. The red arrow indicates the shifted location of the main DM halo located at the red cross. The contour at $1.5 \times 10^{9} M_{\odot} \mathrm{kpc}^{-2}$ guides the eye to the apparent comet-like profile as seen in the X-ray luminosity distribution reported by previous studies (Ebeling et al. 2014; Parekh et al. 2017; Sandhu et al. 2018). A direct comparison between the DM and X-ray light distributions is shown and discussed in Jauzac et al. (2019). The projected mass density distributions are similar between the models in areas north of the BCG, and their contours are virtually indistinguishable around the BCG where the mass distribution is well constrained. The main differences between the models appear in the southeast, due to the lack of constraints in that side of the cluster (see Section 3.3 for more details).

We report an effective Einstein radius of $\theta_{E} \simeq 36^{\prime \prime}$ for a source at $z=9$. The effective Einstein radius is the radius of a circle with the same area as an ellipse fitted to the critical curve. We measure a total projected mass of $M_{(200 \mathrm{kpc})}=1.78_{-0.03}^{+0.01} \times$ $10^{14} M_{\odot}$ within $200 \mathrm{kpc}$. Figure 3 shows the radial mass profile centered on the BCG. Using the capability of our parametric approach we compute the mass profile of five different components of our cluster model: the main cluster-scale DM halo, the halos centered on the three BCGs, and the mass distribution of all the other galaxies, which follow a mass-tolight relation.

We qualitatively report a mass ratio of order 100:1 between the main cluster halo and the mass associated with the light of cluster elliptical galaxies, excluding the three brightest galaxies (dark green and magenta lines in Figure 3). This is consistent with the relative mass to light ratio of rich clusters of about $10^{14} M_{\odot}$ as reported in Girardi et al. (2002). One will see that this qualitative result has no uncertainties attached, since the statistical uncertainties of the mass profile in Section 4 are likely underestimating the true uncertainty due to modeling assumptions (e.g., Meneghetti et al. 2017 and structure along the line of sight; Chirivì et al. 2018).

\subsection{Photometric Redshifts}

The lens modeling procedure left the redshift of multiply imaged systems with no spectroscopic confirmation free to vary. It explores the parameter space to find the most likely redshift (model-z) of each system. Generally, we find that the redshifts predicted by the "Silver" model are in agreement with those predicted by the "Bronze" model. However, a comparison between the lens model-predicted redshifts (model-z) and photometric redshift (photo- $z$ ) estimates can be used for a qualitative assessment of the validity of the lens model.

The RELICS program delivered photometric redshift catalogs using BPZ (Benítez 2000; Coe et al. 2006) based on HST photometry measured in ACS and WFC3 images. We compare our model- $z$ results against photometric redshifts from the public catalog, as well as against a photometric redshift analysis that supplements the HST data with Spitzer photometry and uses a different algorithm, EAZY (Brammer et al. 2008). A thorough description of the HST+Spitzer $z_{\text {phot }}$ analysis will be provided in a forthcoming paper (Strait et al. 2019, in preparation).

The multiplicity (i.e., having multiple images for each lensed source) provides an additional means to test the robustness of the photometric redshifts of the lensed galaxies in this field. We note that in some cases, the photo- $z$ measured for the different multiple images of the same source can disagree. That happens even for systems where the visual identification of the multiple images is entirely unambiguous. This discrepancy could be due to contamination from nearby sources (usually cluster members); variations in SExtractor's detection, deblending, and segmentation for each multiple image; and photo- $z$ degeneracies, especially when the lensed image is very faint (mag $>28)$. Structure along the line of sight could potentially increase the uncertainty of the model $-z$, and contribute to a discrepancy between model- $z$ and photo- $z$. However, this effect is likely not significant, and not the main source of discrepancy (Chirivì et al. 2018).

For these reasons, it is instructive to examine the entire probability distribution function (PDF) of the photo- $z$ and model- $z$ when assessing the agreement between them. We show them in Figure 4.

Ruling out photo- $z$ solutions that place securely identified lensed galaxies in front of the cluster, we find that the model- $z$ PDF of most of the sources are in good agreement with the photo- $z$ PDF of at least one of the multiple images of that source. However, we note a discrepancy between the model- $z$ and the photo- $z$ in the case of some of the sources and discuss it in the following paragraph.

The most problematic discrepancy is for source 9. The HST colors and both the HST and HST+Spitzer $z_{\text {phot }}$ PDFs rule out redshifts above 6 , and the photo- $z$ solutions of the two different images of the same source are in agreement. However, when the redshift of this system is set as a free parameter with a flat prior and no upper limit, all the lens models, including the "bridge" model, favor an extremely high redshift $(z \sim 9)$, albeit with large uncertainty. System 9 is a pair of images that closely straddle the critical curve. Such systems, if their spectroscopic redshift is known, can be excellent constraints, since they tightly constrain the location of the critical curve. On the other hand, when the redshift of such a pair is unknown, only the position of the critical curve is constrained but not its redshift. Based on the colors and photo- $z$ estimates for this source, we rule out the $z \sim 9$ solution. To examine the effect of this wrong solution on the lens model results, we computed a separate model with the redshift of system 9 fixed at $z=5.75$, the most probable photo- $z$ of image 9.1 from the $H S T+$ Spitzer EAZY 


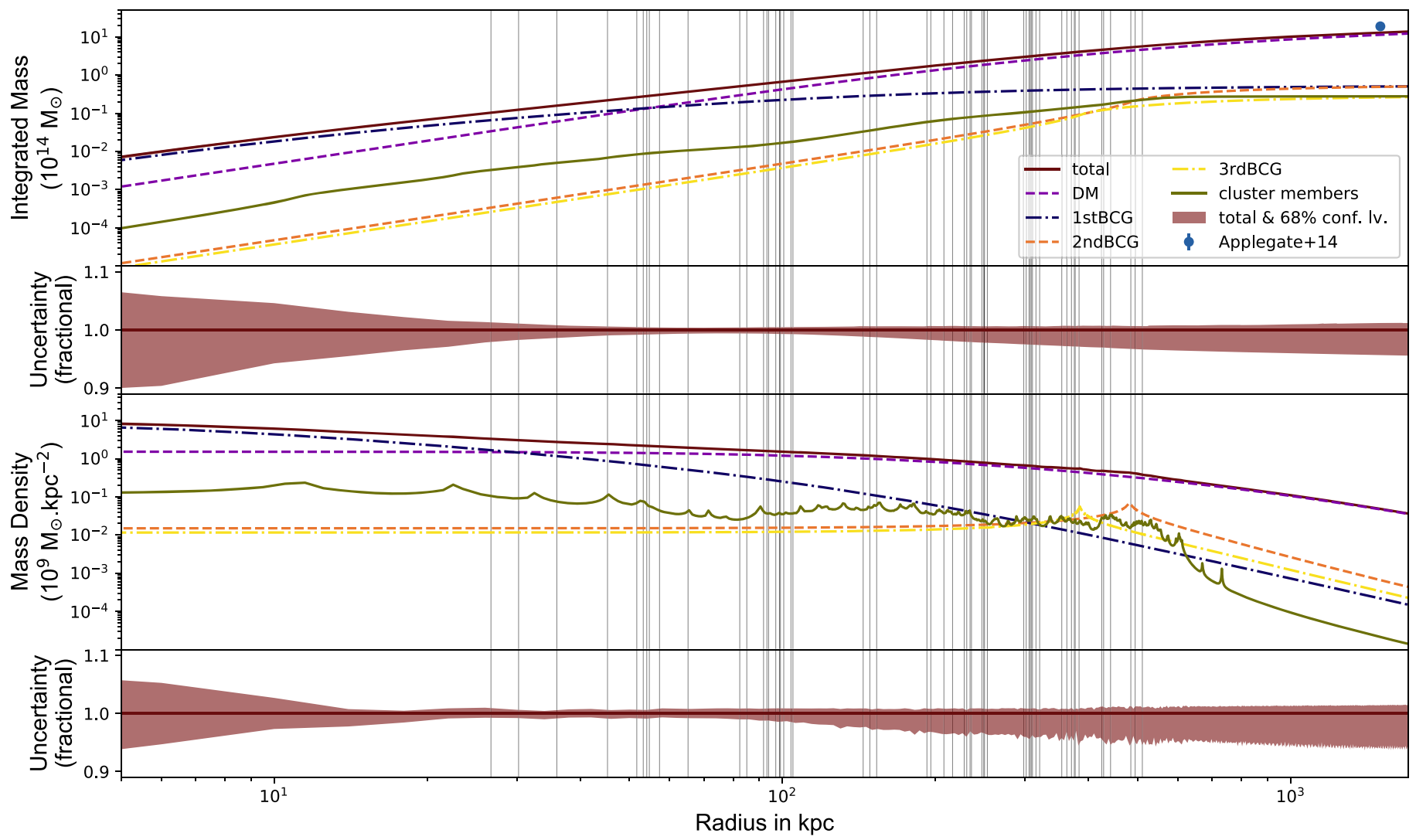

Figure 3. Top: integrated mass profiles within a circular aperture centered on the BCG. Our parametric approach enables us to separate the different components of our mass profile. The profile labeled total represents our best fiducial model (i.e., using gold and silver constraints). The profile labeled DM represents the cluster-scale dark matter halo component (see Section 3.3). The profiles labeled 1stBCG, 2ndBCG, and 3rdBCG show the contribution of the three dark matter potentials placed at the locations of the three brightest galaxies of the cluster. The profile labeled cluster members represents the contribution of all cluster-member galaxies excluding the brightest three. We find a ratio between the main dark matter halo and the cluster members dark matter halo of about 100:1. Strong lensing constraints are plotted as vertical gray lines at their projected distance from the BCG. This is done to highlight where lensing constraints are available. In the regions where multiple images are not identified, the mass profile is an extrapolation. Weak lensing mass measurement from Applegate et al. (2014) is plotted as a blue symbol. Bottom: mass density profiles as a function of the distance to the cluster center. The color coding follows the one from the top panel. The dark-red shaded areas show the $68 \%$-confidence interval statistical uncertainty for the total mass profile, with the fractional error shown below each panel. We note that the small statistical uncertainties derived from the modeling underestimate the true error, which is driven by systematic uncertainties.

photo- $z$ analysis. ${ }^{26}$ The outputs of the resulting model are not significantly different from models that leave this parameter free. Motivated by this examination, in our final model, we set the upper limit of the redshift of system 9 to $z \leqslant 6$.

The model predicted redshifts of sources 14 and 16 show a discrepancy with the HST BPZ PDF; however, the HST+Spitzer photo- $z$ increases the likelihood at higher redshifts, and their probability distributions do not rule out the model-z. Moreover, system 14 is faint (mag $\sim 28-29)$ and classified as bronze, making this disagreement less concerning.

For source 7 , both photo- $z$ analyses favor higher redshift solutions for this source, $z>3.5$, while the model- $z$ converges to $z \sim 2.2$. The region in which this source appears is well constrained by images of sources 12 and 13 . For 7.1 and 7.2 to be multiple images of the same source, source 7 must be at lower redshift than sources 12 and 13. If the photo- $z$ is correct, this source may be misidentified, as already suggested by its classification as bronze.

The photo- $z$ PDFs of several systems, including systems 8 and 17 on the opposite side of systemas 9 and 14, indicate several solutions spanning a large range. Some of these

\footnotetext{
${ }^{26}$ The $H S T$ BPZ analysis yields $z_{\text {phot }} \sim 5.4$, thus this galaxy was not included as a high- $z$ candidate in Salmon et al. (2017).
}

solutions favor a higher redshift than predicted by the lens model. However, we cannot make definitive conclusions for such systems.

Finally, we note that the photometric redshifts that were estimated from the HST data alone were calculated using the BPZ algorithm, and HST+Spitzer photometric redshifts were calculated with EAZY. While a thorough comparison of photometric redshifts is beyond the scope of this paper (e.g., Salmon et al. 2017), we show in Figure 8 in Appendix B a similar comparison using the EAZY algorithm for both the HST and HST+Spitzer photometric redshifts.

\section{High-redshift Predictions}

During the first year of JWST science operations, at least 13 galaxy clusters will be observed in the context of the Guaranteed Time Observations (GTO) and Director's Discretionary Early Release Science (DD-ERS) programs (PIs: Windhorst, Willott, Stiavelli, Rigby, and Treu) using all four JWST instruments: the Near-Infrared Camera (NIRCam), the Near-Infrared Imager and Slitless Spectrograph (NIRISS), Near-Infrared Spectrograph (NIRSpec), and the Mid-Infrared Instrument (MIRI). These observations will include NIRCam imaging to various depths for all 13 clusters. MACS J0417.5-1154 is included in this list of 

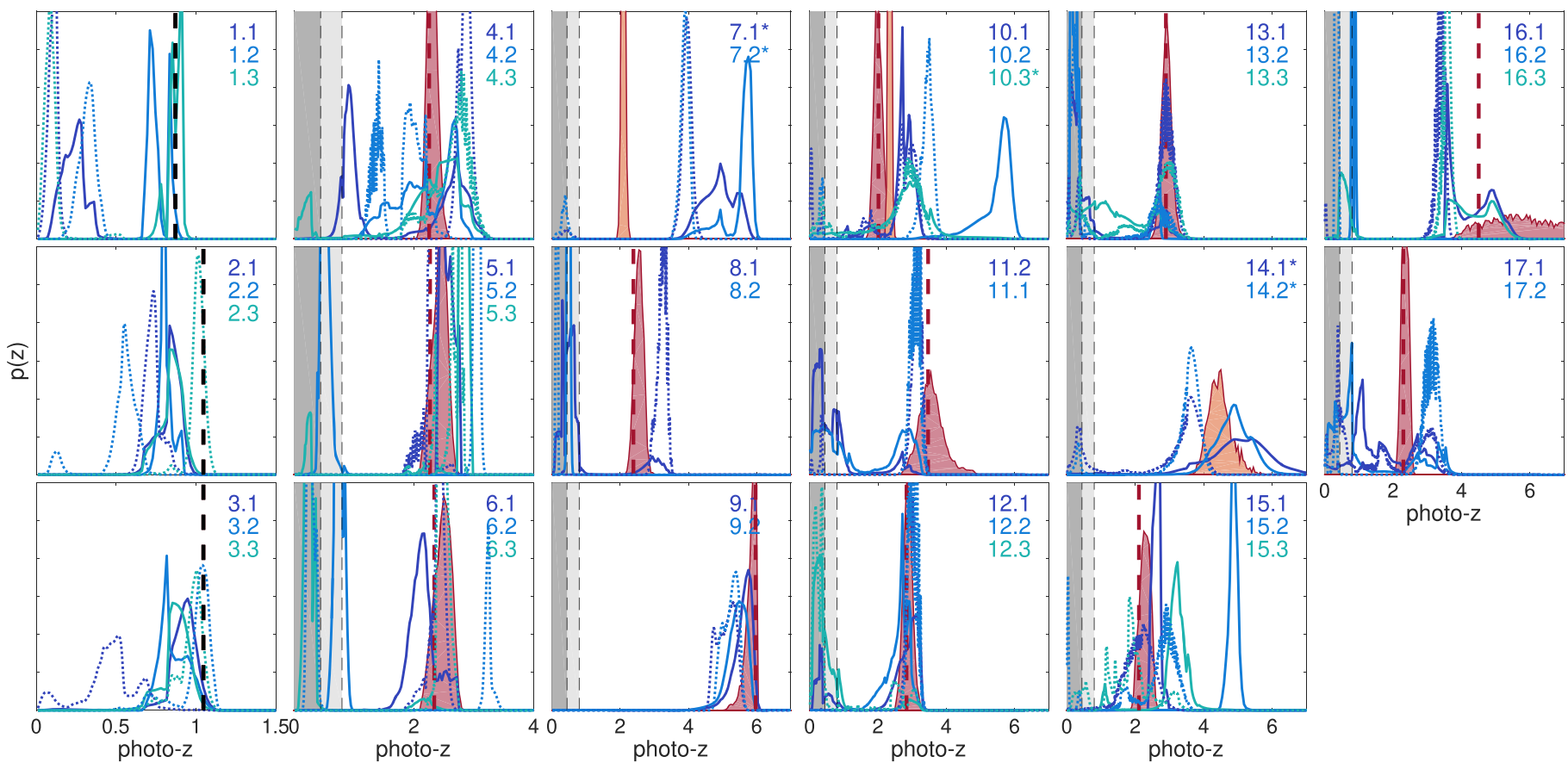

Figure 4. Redshift probability distribution functions (PDFs) of the multiply imaged galaxies used as constraints in the lensing analysis. The blue lines represent photometric redshift PDF estimates from BPZ using the seven HST bands (dotted lines) and from EAZY using the seven HST and two Spitzer bands (solid lines). The red shaded distributions are our lens model estimates based on MCMC sampling of the parameter space. The red vertical dashed lines show the best-fit value, model- $z$, for each system. The light orange shaded areas show predictions from the fiducial ("silver") lens model for multiple images not included in the "silver" set: the bronze systems, 7 and 14, and system 10 with its third counter image 10.3 is included. Systems 1, 2, and 3 have a measured spectroscopic redshift shown as vertical black dashed lines. The dark gray shaded area marks the redshift range in front of the cluster $(z<0.443)$. The light gray shaded area marks the redshift range $0.443<z<0.8$, for which sources $4-17$ would not be strongly lensed. The numbers in each panel correspond to the multiple image identification numbers as reported in Figure 1 and Table 2. An asterisk marks the bronze galaxies. See Section 4 for more detail.

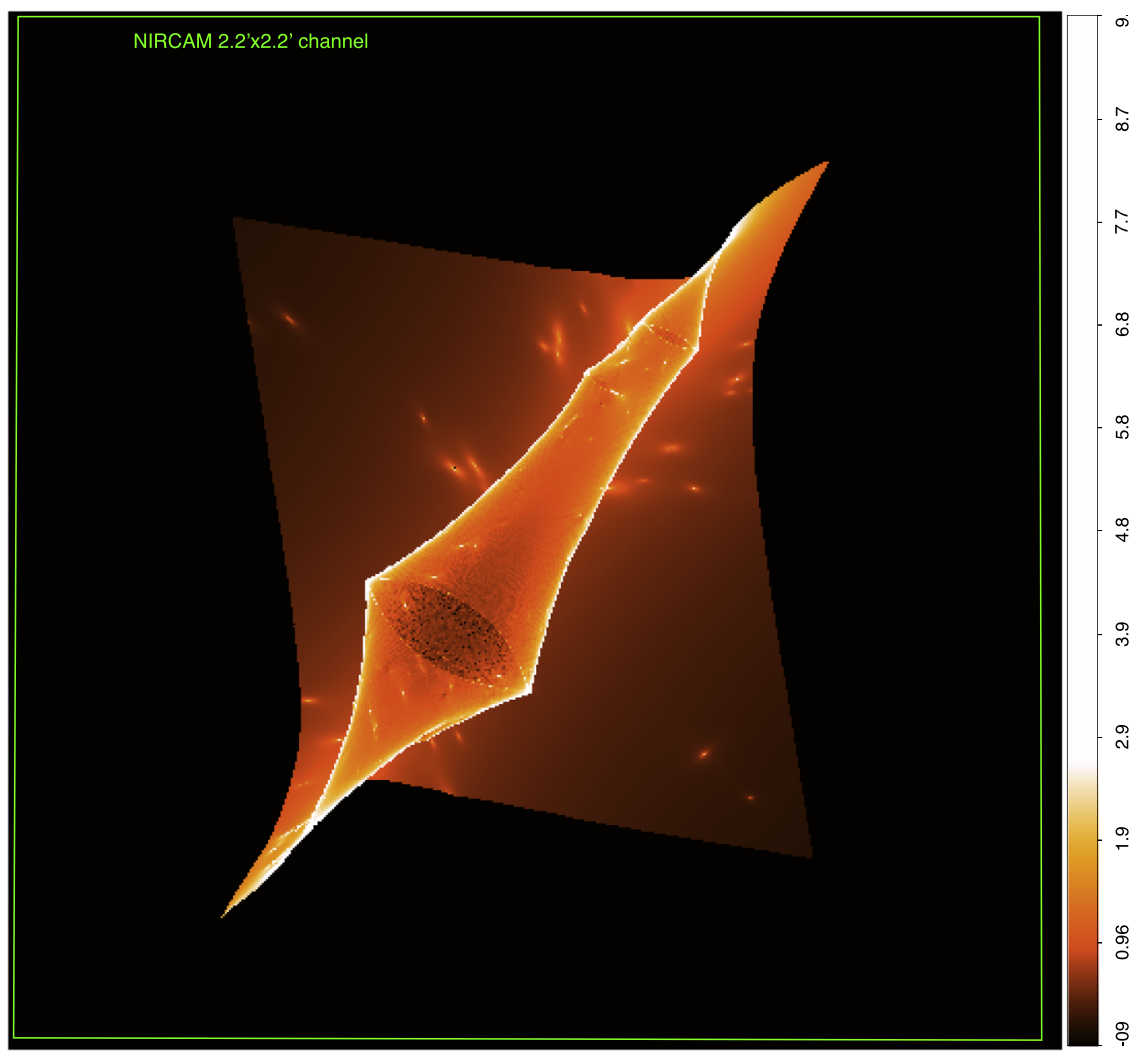

Figure 5. Delensed image of the MACS J0417 magnification map for sources at $z=16$, showing the source-plane area $\left(1.3 \operatorname{arcmin}^{2}\right)$ lensed into a 2 '. $\times 2$ '. field of view $\left(4.8 \mathrm{arcmin}^{2}\right)$, field of view covered by a single NIRCam module. The color scale shows the magnification factor in magnitudes. Beyond $z=7$, the delensed map does not differ significantly from the one presented here. 


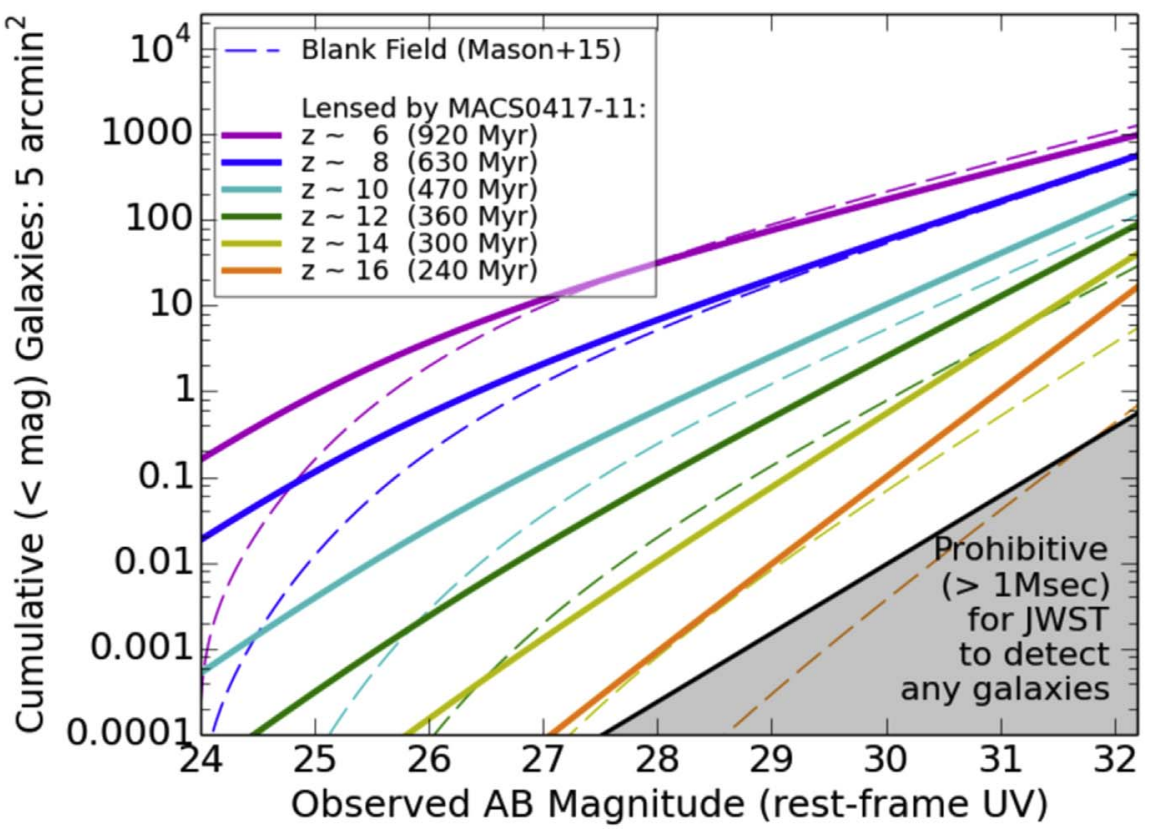

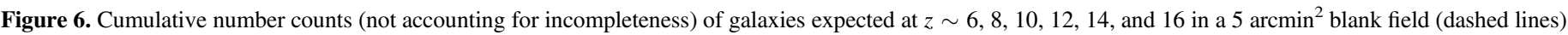

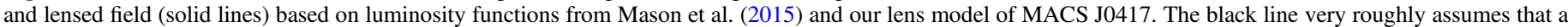

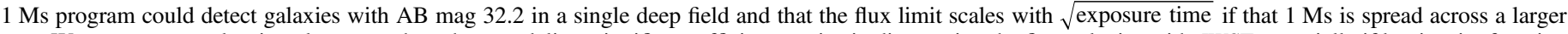

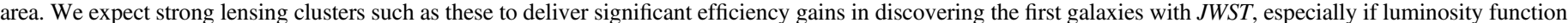
faint-end slopes are as steep as predicted by Mason et al. (2015).

13 clusters and will be observed thanks to the Canadian NIRISS Unbiased Cluster Survey GTO program (CANUCS; PI: Willott).

We use our lens model and UV luminosity functions from Mason et al. (2015) to predict numbers of objects observable by JWST at $8<z<16$, before and during the epoch of reionization. We also explore and discuss the expectations from the HST RELICS observations that yielded 321 candidates with photometric redshifts $z_{\text {phot }} \sim 6-8$ in 46 cluster fields, but none from this cluster (Salmon et al. 2017).

Observing the high-redshift universe behind a cluster offers a boost in sensitivity to lower luminosities, but diminishes the FOV. In Figure 5, we demonstrate how the effective observed FOV of $2 ! 2 \times 2 ! 2\left(4.8 \operatorname{arcmin}^{2}\right.$, or one of the two modules observed by the JWST/ NIRCam), is affected by gravitational lensing. The magnification map for a source at $z=16$ is raytraced through the best-fit model to the source plane. This transformation reveals the spatial extent of the background area covered by such an observation, resulting in an unlensed observed high- $z$ area of $1.3 \operatorname{arcmin}^{2}$.

Figure 6 shows the expected cumulative number counts (not accounting for incompleteness) for MACS J0417, or a galaxy cluster with similar lensing strength, as a function of magnitude, for galaxies at $z=6,8,10,12,14$, and 16 within the FOV of a single NIRCam module (roughly aligned with the WFC3/IR FOV). We adopt blank field luminosity functions from Mason et al. (2015) due to its ability to predict density at any redshift. The faint-end slope of this luminosity function increases from $\alpha=-2.1$ at $z=8$ to $\alpha=-3.5$ at $z=16$. Such steep faint-end slopes would imply that many small, faint galaxies are magnified into view by lensing and that there is a significant efficiency gain from strong lensing to discover the first galaxies with JWST.
Cluster observations scheduled for the first year of JWST will typically reach magnitudes of $\sim 29 \mathrm{AB}$ and fainter. From Figure 6, we expect that at this magnitude limits this field hosts three lensed galaxies at $z=10$, and less than one galaxy in each of the higher redshift bins, not accounting for detection efficiency and incompleteness. Observing an order of a dozen clusters should yield galaxies as distant as $z=12$ and a substantial sample of high- $z$ galaxies at the epoch of reionization.

In Figure 7, we compare the lensing strength of MACS J0417 to other clusters from the RELICS program for which lens models are publicly available on the MAST, including those published by Cerny et al. (2018); Acebron et al. (2018a, 2018b); Cibirka et al. (2018), and Paterno-Mahler et al. (2018). The previous version of MACS J0417 lens model, V1, available on the MAST, predicts $\sim 20 \%$ higher number counts for relatively bright sources (AB mag 25), and similar number counts for faint sources, giving an indication of the systematic uncertainties due to spectroscopic redshift availability, and different modeling assumptions.

With the updated model (V2), we find that MACS J0417 is ranked in the lower 25th percentile of these clusters when it comes to the lensing strength. However, as other RELICS clusters, MACS0417 is among the most powerful lenses known to date.

In a photometric search for $z \sim 6-8$ galaxies in the entire RELICS survey, Salmon et al. (2017) report 321 candidates, with a median of six candidates per field and an average of seven, none of which are in the field of MACS J0417. From Poisson statistics alone, there is a $4 \%$ chance that at least 1 of the 46 RELICS fields would yield no $z \sim 6-8$ candidates, given the average of seven per field. Cosmic variance would increase this likelihood somewhat (Trenti \& Stiavelli 2008), 


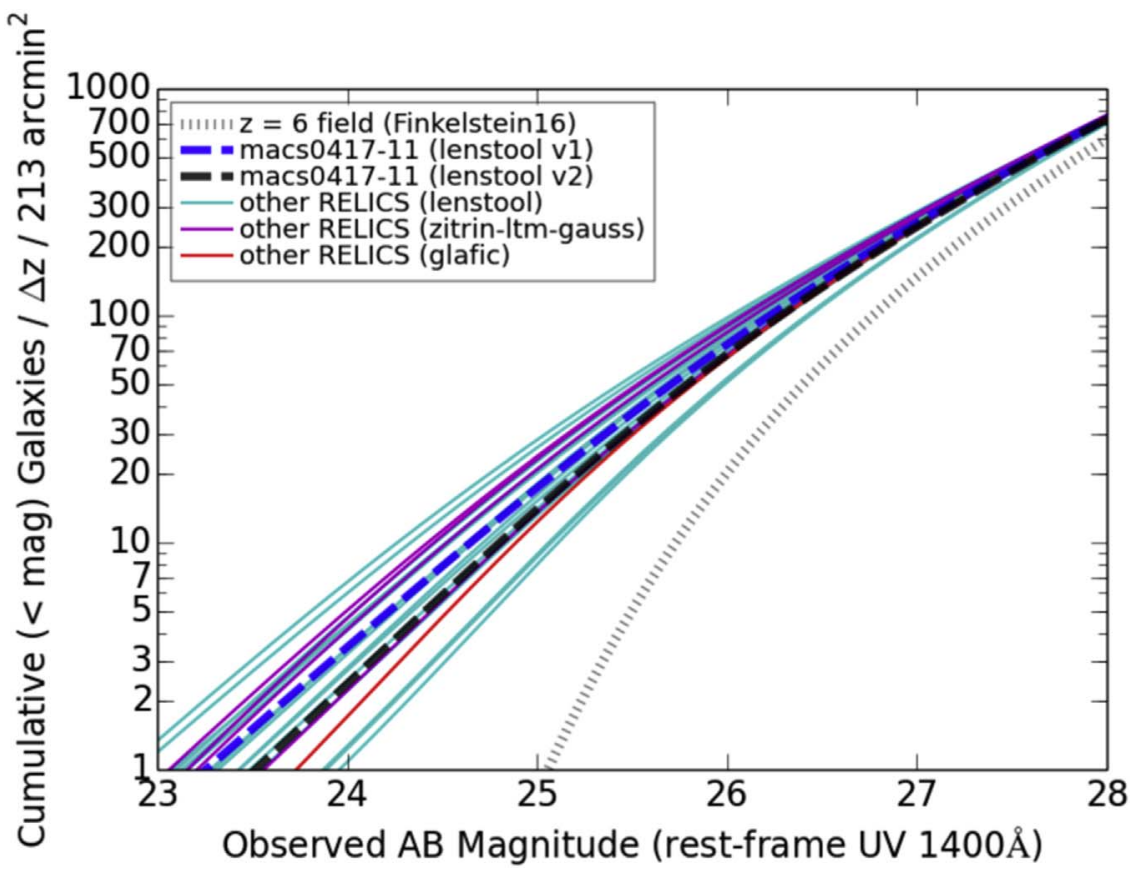

Figure 7. Expected number counts (not accounting for incompleteness) of $z=6$ galaxies in blank fields (dashed line) or lensed by RELICS clusters according to our models (solid lines). The first RELICS lens model (V1; dark blue line) predicts MACS J0417 to have a relatively average lensing strength compared to other RELICS clusters. On the other hand, the model presented in this paper (V2; black line) is among the $25 \%$ weakest of 21 RELICS clusters for which mass models are already available. All expectations are scaled to the full area of $213 \mathrm{arcmin}^{2}$ covered on the sky by RELICS. The publicly available lens models were derived with various methods: Lenstool (Kneib et al. 1996; Jullo et al. 2007), Zitrin-LTM (Broadhurst et al. 2005; Zitrin et al. 2015), and GLAFIC (Oguri 2010).

especially in a lensed field (Robertson et al. 2014). However, our lensing analysis indicates that the lensing strength of MACS J0417 is not extraordinarily low compared to other RELICS clusters for which models are available. It is therefore odd that Salmon et al. (2017) detected no $z_{\text {phot }} \sim 6-8$ candidates in this field.

Quantitatively, the prediction for MACS J0417, shown in Figure 7, indicates that this field should host about $5.34 z \sim 6$ magnified galaxies at, or brighter than, $27 \mathrm{mag}$. The actual expected number would be lower, due to incompleteness. A thorough investigation, including completeness estimates, is required (e.g., Livermore et al. 2017). However, we can get a rough estimate of the detection efficiency of Salmon et al. (2017) for the discovery of $z \sim 6$ galaxies from their actual detection histograms. Salmon et al. (2017) discovered 211 candidates with F160W AB mag $\leqslant 27$ in the $z_{\text {phot }}=6$ bin in all of the RELICS fields. From Figure 7, we expect there to be at most 300 galaxies at $z=6$ with observed $\mathrm{AB}$ magnitude below 27 within the same observed area. A comparison of the number of candidates observed with the predicted number implies an estimated average efficiency of at least $70 \%$. Assuming this efficiency, we would have expected Salmon et al. (2017) to find at least $5.34 \times 70 \%=3.74$ galaxies in this range behind MACS J0417. Assuming small number statistics, the zero detection is discrepant with this estimated expectation (for example, Poisson statistics would give a range of 1-8 at $95 \%$ confidence level). The low number of candidates in this field could be a result of lower-than-average density of galaxies at this location due to cosmic variance. However, such discrepancy suggests a reanalysis of this particular field is needed.

As can be seen in Figures 3 and 8, some of the EAZY photo- $z$ PDFs favor $z>5.5$ solutions for some of the multiple images. A preliminary BPZ reanalysis of this field puts source 9 slightly above $z_{\text {phot }}=5.5$, which would increase the number of candidates in this field to two $z \sim 6$ candidates. Therefore, reducing the disagreement between predictions and detections.

An analysis of this field and all RELICS fields based on the combined $H S T+$ Spitzer photometry is in progress (V. Strait et al. 2019, in preparation). Adding the Spitzer photometry could remove some of the degeneracies and improve the photometric redshift estimates.

\section{Discussion and Summary}

We present a strong lens model of MACS J0417.5-1154, updating the model previously released by the RELICS collaboration. This cluster was selected by the RELICS program for its promising lensing capabilities. We identified 57 multiple images belonging to 17 lensed background sources. We also report lensing candidates that were not reliable enough to be used as constraints but are nevertheless of potential interest for further study by current or upcoming facilities such as JWST. This study and the companion paper by Jauzac et al. (2019) represent the first published strong lensing analyses of this cluster.

Our strong lensing analysis compares models based on constraints with different levels of reliability (silver and bronze) as well as different levels of complexity of the lens plane, for example when including a bridge of matter between the two main substructures of the cluster. Our analysis reveals that the addition of a bridge potential, while giving a lower rms does not satisfy our BIC criteria. Therefore, we keep a fiducial model constrained by our silver sample with no potential acting as a bridge of matter between substructures of the cluster.

From our strong lensing mass modeling, we measure a total projected mass within $200 \mathrm{kpc}$ of $M_{(200 \mathrm{kpc})}=1.78_{-0.03}^{+0.01} \times$ 

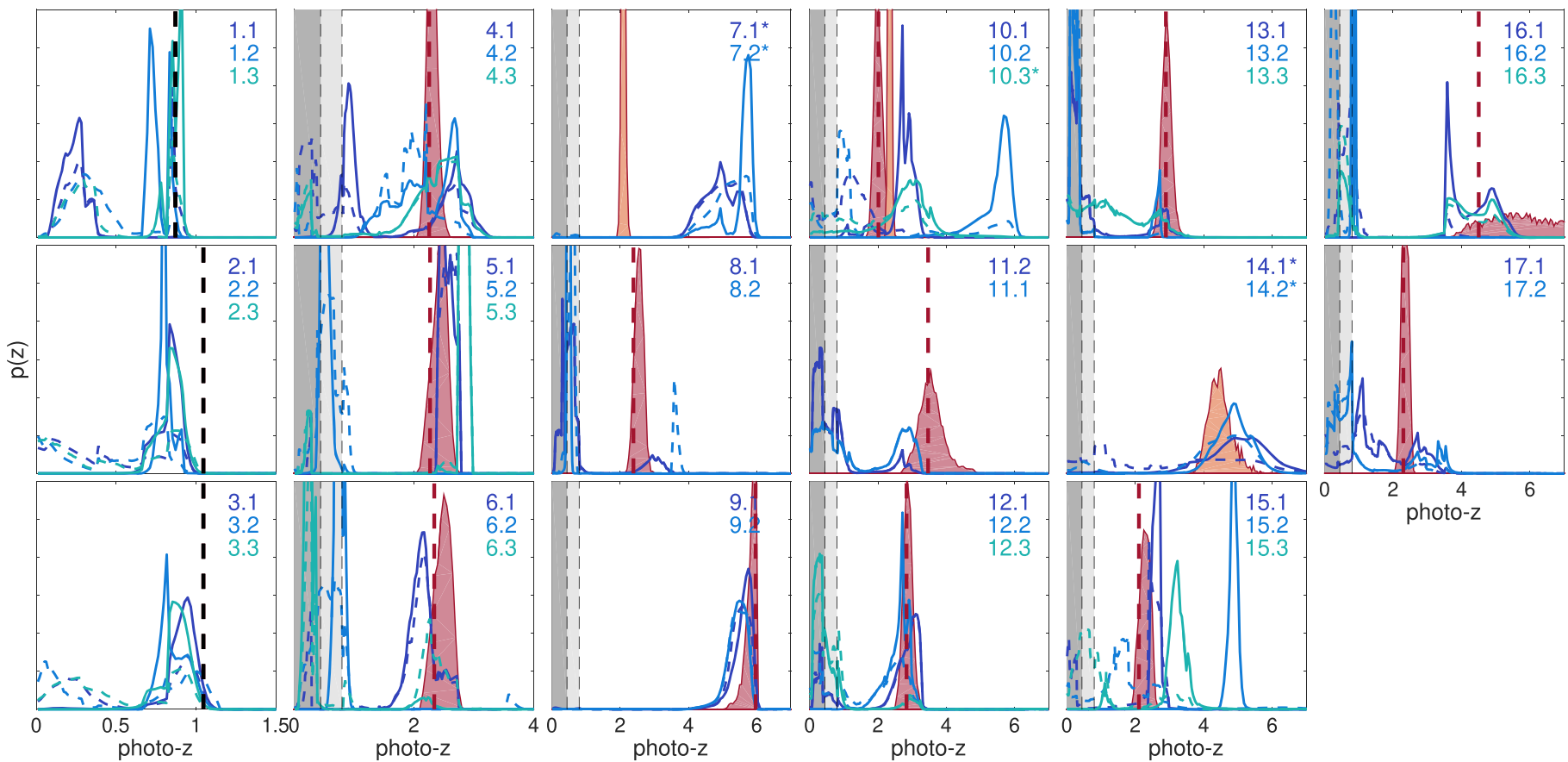

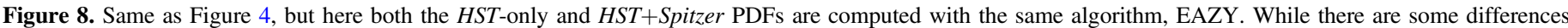
between the EASY and BPZ outputs, choosing one algorithm over the other does not change the results of this paper.

$10^{14} M_{\odot}$. Using the parametric capability of our modeling we estimate the mass ratio between the large-scale halo and the galaxy halos to be of order 100:1. Extrapolating the mass model to a large projected radius, we find a mass at $1.5 \mathrm{Mpc}$ of $M_{(1.5 \mathrm{Mpc})}=12.88_{-0.51}^{+0.16} \times 10^{14} M_{\odot}$. Despite the limited ability of strong lens models to measure the mass beyond the multiple image region, this value is within $3 \sigma$ of the mass $M_{1.5 \mathrm{Mpc}}=$ $(18.9 \pm 0.25) \times 10^{14} M_{\odot}$ measured by weak lensing analysis (Applegate et al. 2014). We report for this cluster an Einstein radius of $\theta_{E} \simeq 36^{\prime \prime}$ at $z=9$. Using the parameters of the spherical Navarro-Frenk-White (NFW; Navarro et al. 1997) profile fitted in Applegate et al. (2014), we derived an Einstein radius at $z=9$ of $\theta_{E_{\mathrm{NFW}}} \simeq 26^{\prime \prime}$. The large mass reported by Applegate et al. (2014) still provides a reasonably close Einstein radius compare to our analysis. In addition, strong lensing analysis of CLASH clusters (Zitrin et al. 2015) report, for comparable clusters, similar values.

We examine the agreement between photo- $z$ and model- $z$ for the sample of multiple images selected in our study. There is a general agreement when the low $z$ solutions for the photo- $z$ are excluded. System 7 might be a misidentification. The agreements for systems 12 and 13 benefit from the reduced redshift range during the optimization of system 9 induced by the initial disagreement with photo- $z$. A detailed study of the influence of the photometric redshift algorithm or the data set is beyond the scope of this paper as this would need more spectroscopic redshifts to be used as a benchmark to remove biases in the comparison.

Our previous model of MACS J0417 suggested its lensing strength was about average among all RELICS clusters modeled to date (all of which are powerful lenses). The new lens model presented here suggests MACS J0417 is in the lower 25th percentile of the RELICS clusters. Still the lack of any $z_{\text {phot }} \sim 6-8$ candidates in this field is at odds with the expected number, estimated from the lensing magnification of this field, assumptions on the high- $z$ luminosity functions, and our estimate of the average detection efficiency of Salmon et al. (2017). We primarily attribute this to cosmic variance, but we will reanalyze this field and perform completeness simulations to determine if there are some other reasons besides cosmic variance for the low yield of high- $z$ candidates. MACS J0417 is still expected to be an efficient lens for upcoming JWST GTO observations to discover fainter and higher redshift candidates. Strong lensing clusters will continue to deliver significant efficiency gains toward discovering high-redshift galaxies and the first galaxies with JWST.

Support for program GO-14096 was provided by NASA through a grant from the Space Telescope Science Institute, which is operated by the Association of Universities for Research in Astronomy, Inc., under NASA contract NAS526555. This paper is based on observations made with the NASA/ESA Hubble Space Telescope, obtained at the Space Telescope Science Institute, which is operated by the Association of Universities for Research in Astronomy, Inc., under NASA contract NAS 5-26555. These observations are associated with program GO-14096. Archival data are associated with program GO-12009. This paper includes data gathered with the $6.5 \mathrm{~m}$ Magellan Telescopes located at Las Campanas Observatory, Chile. M.J. was supported by the Science and Technology Facilities Council (grant No. ST/ L00075X/1) and used the DiRAC Data Centric system at Durham University, operated by the Institute for Computational Cosmology on behalf of the STFC DiRAC HPC Facility (www.dirac.ac.uk). This equipment was funded by BIS National E-infrastructure capital grant ST/K00042X/1, STFC capital grant ST/H008519/1, and STFC DiRAC Operations grant ST/K003267/1 and Durham University. DiRAC is part of the National E-Infrastructure. A.C.E. acknowledges support from STFC grant ST/P00541/1. I.U.R. acknowledges support 
from NSF grants AST-1613536 and AST-1815403. Lawrence Livermore National Laboratory is operated by Lawrence Livermore National Security, LLC, for the U.S. Department of Energy, National Nuclear Security Administration under Contract DE-AC52-07NA27344

Facilities: HST(WFC3, ACS) Magellan(LDSS3), VLT(MUSE).

\section{Appendix A Candidate Multiple Images}

We provide a list of candidate multiple images that were discovered in this work. These galaxies were not deemed reliable enough to be used as constraints. If confirmed with deeper observations, they could become useful lensing evidence to constrain areas in the field that are currently under-constrained. Table 4 lists the candidate IDs and coordinates. They are plotted in Figure 1.

\section{Appendix B EASY Photo- $z$ Estimates}

The photo- $z$ estimates that were used in this analysis are computed with two different algorithms, the HST-only analysis was done with BPZ and matches the catalogs that are publicly available on MAST. The HST+Spitzer analysis uses EAZY. In this appendix, we repeat the comparison between modelpredicted redshift PDFs and those of the photo- $z$ estimates, using EASY for both sets of photo- $z$ measurements (see Figure 8). The same HST photometric catalogs, also available on MAST, were used in all cases. We find that the choice of photo- $z$ algorithm does not significantly change our conclusion that the photometric redshifts and model redshifts are generally in good agreement.

\section{ORCID iDs}

Guillaume Mahler (iD https://orcid.org/0000-0003-3266-2001

Keren Sharon (iD https://orcid.org/0000-0002-7559-0864

Dan Coe (ib https://orcid.org/0000-0001-7410-7669

Victoria Strait (i) https://orcid.org/0000-0002-6338-7295

Alastair Edge (iD https://orcid.org/0000-0002-3398-6916

Ana Acebron (iD https://orcid.org/0000-0003-3108-9039

Felipe Andrade-Santos (iD https://orcid.org/0000-0002-8144-9285

Maruša Bradač (iD https://orcid.org/0000-0001-5984-0395

Larry D. Bradley (iD https://orcid.org/0000-0002-7908-9284

Daniela Carrasco (iD https://orcid.org/0000-0002-3772-0330

Nathália Cibirka (i) https://orcid.org/0000-0002-2356-4680

William A. Dawson (ib https://orcid.org/0000-0003-0248-6123

Austin T. Hoag (iD https://orcid.org/0000-0001-8989-2567

Kuang-Han Huang (1) https://orcid.org/0000-0001-7826-6448

Traci L. Johnson (10 https://orcid.org/0000-0002-8829-5303

Daniel Lam (1) https://orcid.org/0000-0002-6536-5575

Rachael Livermore (ib https://orcid.org/0000-0003-4456-1566

Ramesh Mainali (1) https://orcid.org/0000-0003-0094-6827

Masami Ouchi (i) https://orcid.org/0000-0002-1049-6658

Rachel Paterno-Mahler (iD https://orcid.org/0000-0003-

3653-3741

Ian U. Roederer (10 https://orcid.org/0000-0001-5107-8930

Brett Salmon (iD https://orcid.org/0000-0002-7453-7279

Sune Toft (iD https://orcid.org/0000-0003-3631-7176

Michele Trenti (i) https://orcid.org/0000-0001-9391-305X

Keiichi Umetsu (iD https://orcid.org/0000-0002-7196-4822

Benedetta Vulcani iD https://orcid.org/0000-0003-0980-1499

Adi Zitrin (Di) https://orcid.org/0000-0002-0350-4488

\section{References}

Acebron, A., Alon, M., Zitrin, A., et al. 2018b, arXiv:1810.08122

Acebron, A., Cibirka, N., Zitrin, A., et al. 2018a, ApJ, 858, 42

Acebron, A., Jullo, E., Limousin, M., et al. 2017, MNRAS, 470, 1809

Applegate, D. E., von der Linden, A., Kelly, P. L., et al. 2014, MNRAS, 439,48

Atek, H., Richard, J., Kneib, J.-P., et al. 2015, ApJ, 800, 18

Atek, H., Richard, J., Kneib, J.-P., \& Schaerer, D. 2018, MNRAS, 479, 5184

Bacon, R., Accardo, M., Adjali, L., et al. 2010, Proc. SPIE, 7735, 773508

Becker, G. D., Bolton, J. S., \& Lidz, A. 2015, PASA, 32, e045

Becker, R. H., Fan, X., White, R. L., et al. 2001, AJ, 122, 2850

Benítez, N. 2000, ApJ, 536, 571

Bertin, E., \& Arnouts, S. 1996, A\&AS, 117, 393

Bhatawdekar, R., Conselice, C., Margalef- Bentabol, B., \& Duncan, K. 2018, arXiv: 1807.07580

Botteon, A., Gastaldello, F., \& Brunetti, G. 2018, MNRAS, 476, 5591

Bouwens, R. J., Oesch, P. A., Illingworth, G. D., Ellis, R. S., \& Stefanon, M. 2017, ApJ, 843, 129

Bradley, L. D., Zitrin, A., Coe, D., et al. 2014, ApJ, 792, 76

Brammer, G. B., van Dokkum, P. G., \& Coppi, P. 2008, ApJ, 686, 1503

Broadhurst, T., Benítez, N., Coe, D., et al. 2005, ApJ, 621, 53

Cerny, C., Sharon, K., Andrade-Santos, F., et al. 2018, ApJ, 859, 159

Chirivì, G., Suyu, S. H., Grillo, C., et al. 2018, A\&A, 614, A8

Cibirka, N., Acebron, A., Zitrin, A., et al. 2018, ApJ, 863, 145

Coe, D., Benítez, N., Sánchez, S. F., et al. 2006, AJ, 132, 926

Coe, D., Zitrin, A., Carrasco, M., et al. 2013, ApJ, 762, 32

Djorgovski, S. G., Castro, S., Stern, D., \& Mahabal, A. A. 2001, ApJL, 560, L5

Dressler, A., Bigelow, B., Hare, T., et al. 2011, PASP, 123, 288

Dwarakanath, K. S., Malu, S., \& Kale, R. 2011, JApA, 32, 529

Ebeling, H., Edge, A. C., \& Henry, J. P. 2001, ApJ, 553, 668

Ebeling, H., Stephenson, L. N., \& Edge, A. C. 2014, ApJL, 781, L40

Elíasdóttir, Á, Limousin, M., Richard, J., et al. 2007, arXiv:0710.5636

Fan, X., Strauss, M. A., Becker, R. H., et al. 2006, AJ, 132, 117

Girardi, M., Manzato, P., Mezzetti, M., Giuricin, G., \& Limboz, F. 2002, ApJ, 569,720

Gladders, M. D., \& Yee, H. K. C. 2000, AJ, 120, 2148

Green, T. S., Edge, A. C., Stott, J. P., et al. 2016, MNRAS, 461, 560

Gunn, J. E., \& Peterson, B. A. 1965, ApJ, 142, 1633

Hashimoto, T., Laporte, N., Mawatari, K., et al. 2018, Natur, 557, 392

Ishigaki, M., Kawamata, R., Ouchi, M., et al. 2018, ApJ, 854, 73

Jauzac, M., Harvey, D., \& Massey, R. 2018, MNRAS, 477, 4046

Jauzac, M., Mahler, G., Edge, A. C., et al. 2019, MNRAS, 483, 3082

Jauzac, M., Richard, J., Jullo, E., et al. 2015, MNRAS, 452, 1437

Johnson, T. L., \& Sharon, K. 2016, arXiv:1608.08713

Jullo, E., Kneib, J.-P., Limousin, M., et al. 2007, NJPh, 9, 447

Kneib, J.-P., Ellis, R. S., Smail, I., Couch, W. J., \& Sharples, R. M. 1996, ApJ, 471,643

Lagattuta, D. J., Richard, J., Clément, B., et al. 2017, MNRAS, 469, 3946

Limousin, M., Jullo, E., Richard, J., et al. 2010, A\&A, 524, A95

Limousin, M., Kneib, J.-P., \& Natarajan, P. 2005, MNRAS, 356, 309

Livermore, R. C., Finkelstein, S. L., \& Lotz, J. M. 2017, ApJ, 835, 113

Lotz, J. M., Koekemoer, A., Coe, D., et al. 2017, ApJ, 837, 97

Mahler, G., Richard, J., Clément, B., et al. 2018, MNRAS, 473, 663

Mann, A. W., \& Ebeling, H. 2012, MNRAS, 420, 2120

Mason, C. A., Trenti, M., \& Treu, T. 2015, ApJ, 813, 21

Meneghetti, M., Natarajan, P., Coe, D., et al. 2017, MNRAS, 472, 3177

Merlin, E., Fontana, A., Ferguson, H. C., et al. 2015, A\&A, 582, A15

Merlin, E., Fontana, A., Ferguson, H. C., et al. 2016, T-PHOT: PSF-matched, Prior-based, Multiwavelength Extragalactic Deconfusion Photometry, Astrophysics Source Code Library, ascl:1609.001

Navarro, J. F., Frenk, C. S., \& White, S. D. M. 1997, ApJ, 490, 493

Oemler, A., Clardy, K., Kelson, D., Walth, G., \& Villanueva, E. 2017, COSMOS: Carnegie Observatories System for MultiObject Spectroscopy, Astrophysics Source Code Library, ascl:1705.001

Oesch, P. A., Bouwens, R. J., Illingworth, G. D., Labbé, I., \& Stefanon, M. 2018, ApJ, 855, 105

Oguri, M. 2010, PASJ, 62, 1017

Oke, J. B. 1974, ApJS, 27, 21

Pandge, M. B., Monteiro-Oliveira, R., Bagchi, J., et al. 2018, MNRAS, 2802

Parekh, V., Dwarakanath, K. S., Kale, R., \& Intema, H. 2017, MNRAS, 464, 2752

Paterno-Mahler, R., Sharon, K., Coe, D., et al. 2018, ApJ, 863, 154

Planck Collaboration, Adam, R., Aghanim, N., et al. 2016a, A\&A, 596, A108

Planck Collaboration, Ade, P. A. R., Aghanim, N., et al. 2011, A\&A, 536, A7 
Planck Collaboration, Ade, P. A. R., Aghanim, N., et al. 2016b, A\&A, 594, A27

Postman, M., Coe, D., Benítez, N., et al. 2012, ApJS, 199, 25

Remolina González, J. D., Sharon, K., \& Mahler, G. 2018, ApJ, 863, 60

Robertson, B. E., Ellis, R. S., Dunlop, J. S., et al. 2014, ApJL, 796, L27

Salmon, B., Coe, D., Bradley, L., et al. 2017, arXiv:1710.08930

Salmon, B., Coe, D., Bradley, L., et al. 2018, ApJL, 864, L22
Sandhu, P., Malu, S., Raja, R., \& Datta, A. 2018, Ap\&SS, 363, 133 Schwarz, G. 1978, AnSta, 6, 461

Trenti, M., \& Stiavelli, M. 2008, ApJ, 676, 767

Voges, W., Aschenbach, B., Boller, T., et al. 1999, A\&A, 349, 389

Yue, B., Castellano, M., Ferrara, A., et al. 2018, ApJ, 868, 115

Zheng, W., Postman, M., Zitrin, A., et al. 2012, Natur, 489, 406

Zitrin, A., Fabris, A., Merten, J., et al. 2015, ApJ, 801, 44 